\title{
Quasienergy resonance in a dynamic Wannier-Stark ladder
}

\author{
Yuya Nemoto, ${ }^{1}$ Ken-ichi Hino, ${ }^{2,3, *}$ and Nobuya Maeshima ${ }^{2,3}$ \\ ${ }^{1}$ Doctoral Program in Frontier Science, Graduate School of Pure and Applied Sciences, University of Tsukuba, \\ Tsukuba, Ibaraki 305-8573, Japan \\ ${ }^{2}$ Division of Materials Science, Faculty of Pure and Applied Sciences, University of Tsukuba, Tsukuba 305-8573, Japan \\ ${ }^{3}$ Center for Computational Sciences, University of Tsukuba, Tsukuba 305-8577, Japan
}

(Received 25 April 2012; revised manuscript received 17 February 2013; published 15 May 2013)

\begin{abstract}
We examine a continuum effect of a dynamic Wannier-Stark ladder (DWSL) driven by a cw laser-with $F_{a c}$ and $\omega$ as amplitude and frequency, respectively_-by means of an excess density of states (DOS), $\rho^{(e x)}(E)$, closely related to the more familiar DOS and proportional to the lifetime of a resonance state. It is mathematically shown that $\rho^{(e x)}(E)$ is governed by three different physical mechanisms: the single-channel resonance mechanism, the multichannel nonresonance mechanism, and the multichannel resonance mechanism. The last mechanism becomes more important with the increase in $F_{a c}$. The effect of the interchannel interaction is maximized when the ratio of a Bloch frequency to $\omega$, represented as $\eta$, equals unity. In the actual calculations based on the $R$-matrix Floquet theory, it is revealed that, in a large- $F_{a c}$ region, $\rho^{(e x)}(E)$ for $\eta=1$ shows a complicated spectral structure composed of a couple of newly growing peaks, in contrast to $\rho^{(e x)}(E)$ for $\eta=3$ which just shows a monotonic change of a single spectral peak. It is speculated that the pronounced feature of the former spectra is attributed to the Fano-like multichannel resonance mechanism, whereas the feature of the latter case is attributed to the multichannel nonresonance mechanism.
\end{abstract}

DOI: 10.1103/PhysRevB.87.205305

PACS number(s): 78.67.Pt, 42.50.Hz

\section{INTRODUCTION}

As is well known, "quasienergy" in a temporally periodic system is considered to be as significant a constant of motion as "quasimomentum" in a spatially periodic system, where the existence of both quantum numbers is ensured by the Floquet theorem and the Bloch theorem, respectively. The Floquet theory has been applied to diverse physical systems for the studies of driven quantum tunneling ${ }^{1,2}$ and coherent control, ${ }^{3}$ such as electron-doped semiconductor superlattices (SLs) driven by a terahertz wave, ${ }^{4}$ a cold atomic gas in a one-dimensional optical lattice, ${ }^{5}$ an atomic Bose-Einstein condensate,${ }^{6}$ light curved waveguide arrays ${ }^{7-9}$ Cooper pairs in Josephson qubits, ${ }^{10}$ and strongly correlated electron systems. ${ }^{11}$ Analysis based on the quasienergy reveals that these systems are characterized by the important concepts of dynamic localization, coherent destruction of tunneling, and photonassisted tunneling (PAT).

One of the most common systems ensuring the Floquet theorem in condensed-matter physics is the dynamical Wannier-Stark ladder (dynamic WSL, DWSL) in biased semiconductor SLs driven by a temporally periodic laser. ${ }^{12-14}$ This is the prototype of laser-induced phenomena in semiconductor nanostructures to which considerable attention has been attracted in the context of the coherent dynamics and coherent control of electronic states. It is in terms of quasienergy that the fundamental features of the DWSL-PAT, dynamic localization, quasienergy-band deformation due to ac-Zener tunneling (ac-ZT), and so on-are conventionally understood. Such a standpoint will be termed as the conventional quasienergy picture. The concerned quasienergy is provided by solving a Hermitian eigenvalue problem derived from a tight-binding model, in which, as the need arises, a multiple-SL-miniband approximation incorporating the effect of ac-ZT is made. ${ }^{15-17}$
Actually, the ac-ZT becomes more significant with increasing laser intensity, causing a coupling between photon sidebands attributed to different SL minibands. To be more specific, this interaction contributes to an inter-WSL-site coupling (an intersite ac-ZT effect) as well as an intraWSL-site coupling (an intrasite ac-ZT effect). ${ }^{17}$ The intrasite coupling between WSL levels attributed to different SL minibands is successfully incorporated into the theoretical framework based on the conventional quasienergy picture, and the above-mentioned quasienergy-band deformation is revealed. On the other hand, the intersite coupling between the WSL levels inevitably induces a continuum effect which seems unmanageable in the conventional quasienergy picture without any extension from the original recipe. ${ }^{15-17}$

Strictly speaking, the DWSL problem should be dealt with as a problem with open boundary conditions, namely, the scattering problem. ${ }^{18,19}$ The reason for this is obviously understood by making the Kramers-Henneberger transformation ${ }^{20,21}$ of the original tilted-SL-potential flapping temporally with a laser frequency into a photodressed potential, as will be shown later in more detail. ${ }^{22}$ This intersite ac-ZT effect is reminiscent of the dc-ZT effect in WSL, where the WSL levels attributed to different SL minibands are coupled to each other, leading to delocalization of electronic states across several WSL sites; in particular, such delocalization stands out when Zener resonance takes place. ${ }^{23-27}$ While the WSL problem is reduced to just a single-channel scattering problem, the DWSL problem of concern results in a more involved multichannel scattering problem. Due to this continuum effect, DWSL wave functions are nonsquare integrable and the associated quasienergy spectra are continuous. Interchannel couplings that are to play a key role here are provided by off-diagonal components of a ponderomotive interaction, namely, the matrix element associated with the above-mentioned photodressed potential. 
In the scattering problem of the DWSL, quasienergy is considered to be nothing but a parameter given in advance, and hence the conventional quasienergy picture is no longer effective in this situation. Therefore, an alternative framework is required in order to evaluate basic properties of the DWSL in the whole laser-intensity region. For this purpose, an excess density of states (DOS) as a function of the given quasienergy is proposed in the present paper; this is derived from a scattering matrix obtained by solving the concerned multichannel scattering problem. ${ }^{28-31}$ The excess DOS is closely related to the more familiar DOS and the lifetime of a Floquet state. Accordingly, it is expected that the basic properties of the DWSL can be understood in terms of this physical quantity even in the case where the above-mentioned conventional quasienergy picture is difficult to apply. Such a standpoint will be termed hereafter as the excess-DOS picture.

In this paper, the $R$-matrix Floquet theory ${ }^{32,33}$ is applied to the multichannel scattering problem of the DWSL. By virtue of the excess DOS thus calculated, we examine the significance of the continuum effect on the DWSL in the relatively strong laser-intensity region. Here, let the ratio of a Bloch frequency $\Omega=e F_{0} d$ to a laser frequency $\omega$ be denoted as $\eta$, that is, $\eta=$ $\Omega / \omega$, where $e, F_{0}$, and $d$ represent elementary charge, strength of static electric field, and a lattice constant of the original SLs, respectively. As is shown later, we obtain the result that the excess DOS for $\eta=1$ shows a complicated spectral structure, whereas the excess DOS for $\eta=3$ shows monotonic redshift of a single spectral peak. The underlying physics causing such a sharp difference between these two cases is analyzed in a mathematical manner based on the scattering theory. The excess DOS is represented as a sum of contributions from single-channel resonance scattering, multichannel nonresonance scattering, and multichannel resonance scattering, in which the off-diagonal components of a ponderomotive interaction play a decisive role in an ascending order. It is speculated that the above-mentioned pronounced spectral modulation for $\eta=1$ is due to the multichannel resonance scattering, and the monotonic redshift for $\eta=3$ is due to the multichannel nonresonance scattering. Further, we also show the consistency of the present excess-DOS picture with the conventional quasienergy picture in the weaker laser-intensity region, where the multichannel resonance effect would be negligible.

Below, the present situation of the studies of Floquet resonance states in intense laser-electron interactions is briefly described from the methodological point of view, and the relation with the present study of DWSLs is clarified. The practicable methods for the present would be broadly classified into the complex quasienergy method based on non-Hermitian Floquet matrices ${ }^{34,35}$ and the Floquet approach combined with the $R$-matrix theory, ${ }^{32,33}$ though there is the case in which the non-Hermitian Floquet Hamiltonian is partially employed at a certain level of calculations in the $R$-matrix Floquet theory. Both methods have been applied mostly to atomic and molecular systems rather than condensed-matter systems. The complex quasienergy method was originally developed from the conventional quasienergy picture mentioned above in order to cope with a continuum problem. This method was formulated by performing a complex dilatation in the Floquet equations, namely, a complex scaling technique, ${ }^{36,37}$ and by solving the resulting eigenvalue problem on a basis of real $L^{2}$ functions. ${ }^{34,38,39}$ The existence of Floquet resonance of atomic hydrogen was proved with mathematical rigor by virtue of this technique. ${ }^{22}$ However, it was found to be conceptually more appealing to use the true Hamiltonian with a complex basis such as a complex Sturmian basis set in order to represent proper scattering boundary conditions, namely, truly complex scattering boundary conditions. ${ }^{35}$ On the other hand, the $R$-matrix theory is regarded as one of the most powerful tools for multichannel scattering problems because of not only high numerical accuracy, stability, and efficiency, but also practical feasibility and straightforward applicability to rather involved physical systems; ${ }^{33}$ compared with this method, the above-mentioned complex quasienergy method has been applied to relatively simpler atoms and molecules with a couple of electrons.

The study of Ref. 18 was the first study applying the scattering theory to the DWSL for modeled optical SLs, with $\eta$ fractional, by means of the Floquet-Bloch method. Here, the multichannel scattering problem to be solved is reduced to a non-Hermitian eigenvalue problem with Siegert boundary conditions, and a set of complex quasienergy levels corresponding to resonance positions and the associated lifetimes are provided. Thus, this method is considered to be closely related to the complex quasienergy method with a complex basis set. Meanwhile, the above-mentioned $R$ matrix Floquet theory was already applied to the DWSL problem, ${ }^{19}$ illustrating the instability of dynamic localization due to dynamic Fano resonance by employing the excess DOS. Further, this has been applied to the study of a more complicated excitonic Floquet problem of the DWSL. 40,41

The present study is regarded as the further developed study of Ref. 19 toward a comprehensive understanding of the DWSL with $\eta$ integer. In particular, the case of $\eta=1$ is most important because the effect of PAT is maximized. This paper is aimed at revealing detailed physical mechanisms governing the DWSL, since our preceding study ${ }^{19}$ did not delve deeply into this subject. Hereafter, the conventional quasienergy picture is referred to just as the quasienergy picture when compared with the results based on the excess DOS picture; the results obtained in Ref. 18 for $\eta$ being fractional cannot be compared with the present ones.

The remainder of this article is organized as follows. Section II describes the theoretical framework. Section III presents the results and discussions. Finally, Sec. IV presents the conclusion. Further, the Appendix is included as a supplement. Atomic units (a.u.) are used throughout unless otherwise stated.

\section{THEORY}

\section{A. Basic framework}

The theoretical framework based on the $R$-matrix Floquet theory ${ }^{32,33}$ employed in this study is surveyed, since the details of it are already given in the preceding paper. ${ }^{19} \mathrm{We}$ begin with the following equation:

$$
\left[\mathcal{H}(z, t)-i \frac{\partial}{\partial t}\right] \Phi(z, t)=0,
$$


where

$$
\begin{aligned}
\mathcal{H}(z, t)= & p_{z}\left\{\frac{1}{2 m[z+a(t)]}\right\} p_{z}+V[z+a(t)] \\
& +F_{0} z+v(z, t) .
\end{aligned}
$$

Equation (1) with Eq. (2) is the effective-mass equation for the DWSL wave function $\Phi(z, t)$ in the oscillating frame provided by the Kramers-Henneberger transformation; ${ }^{20,21}$ this is a gauge transformation closely related to the acceleration form of an optical dipole interaction. In Eq. (2), $V(z), m(z), p_{z}$, and $F_{0}$ represent the SL confining potential, an effective mass of an electron, a momentum operator along the crystal growth direction $z$, and a static electric field applied in the $z$ direction, respectively. The $z$ dependence of $m(z)$ is attributed to the effective-mass difference between the well region of SLs and the barrier region of SLs. Such $z$ dependence of the effective mass causes an additional part of laser-electron interactions represented by $v(z, t)$; if $m(z)$ were constant, $v(z, t)$ would vanish. The explicit form of this interaction is not necessary here; for more detail, consult Ref. 19.

Further, $a(t)$-given by $a(t)=\alpha \cos \omega t$-represents the position of a classical electron quivering under the cw-laser field, $F_{a c} \cos \omega t$, applied in the $z$ direction at time $t$, with $F_{a c}$ and $\omega$ as an amplitude and a frequency, respectively. Here, $\alpha$ is called the ponderomotive radius corresponding to the excursion amplitude of a classical electron, defined as

$$
\alpha=\frac{F_{a c}}{m_{a s} \omega^{2}} .
$$

It is assumed that in the asymptotic region of the $z$ direction, namely, $|z+a(t)| \geqslant L_{S L} / 2, \quad V[z+a(t)]$ and $m[z+a(t)]$ become the constant values of $V_{a s}$ and $m_{a s}$, respectively, where $L_{S L}$ represents the size of SL; $V_{a s}$ and $m_{a s}$ are defined to be equal to the barrier height of $V(z)$ and an electron mass in the barrier region of $V(z)$, respectively, following Ref. 19 .

Because of the temporal periodicity of $\mathcal{H}(z, t)$, namely, $\mathcal{H}(z, t+T)=\mathcal{H}(z, t)$, with $T=2 \pi / \omega$, the Floquet theorem allows $\Phi(z, t)$ to be expressed as

$$
\Phi(z, t)=\exp (-i E t) \sum_{\nu=-\infty}^{\infty} \exp (i v \omega t) \psi_{\nu}(z),
$$

where $E$ represents quasienergy. Thus, Eq. (1) is recast into the coupled equations

$$
\sum_{\nu=-\infty}^{\infty}\left[L_{\mu \nu}(z)-E \delta_{\mu \nu}\right] \psi_{\nu}(z)=0,
$$

where $L_{\mu \nu}(z)$ is given by

$$
L_{\mu \nu}(z)=\mathcal{H}_{\mu \nu}(z)+\mu \omega \delta_{\mu \nu} .
$$

Hereafter, the matrix element represented as $[\cdots]_{\mu \nu}$ is defined as

$$
[\cdots]_{\mu \nu}=\frac{1}{T} \int_{0}^{T} \exp [-i(\mu-v) \omega t][\cdots] d t .
$$

Hence, $\mathcal{H}_{\mu \nu}(z)$ is the time average of Eq. (2) in terms of the above expression.

It is noted that the ponderomotive interaction $V_{\mu \nu}(z)$ and the mass-coupling term $[1 / m]_{\mu \nu}(z)$ are functions of $\alpha$ as well as $z$. As regards $V_{\mu \nu}(z)$, for a small $\alpha$, just the diagonal

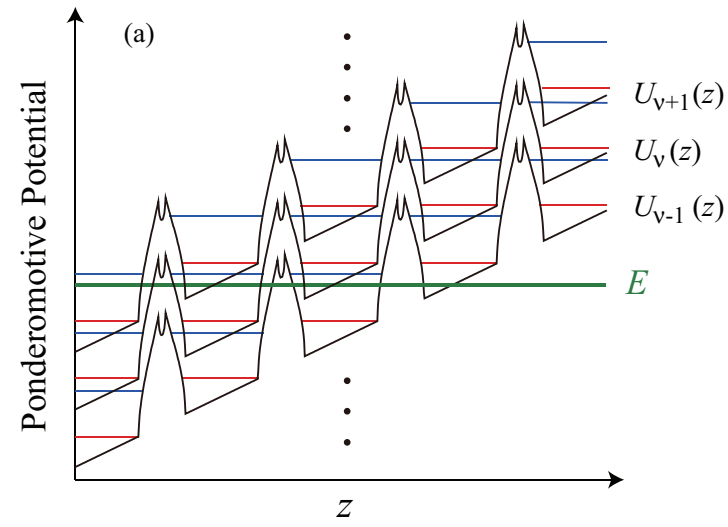

(b)

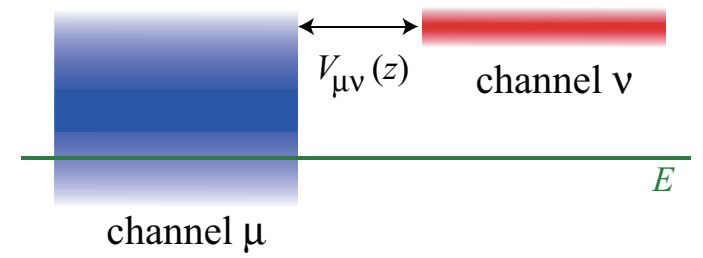

FIG. 1. (Color online) (a) Schematic representation of the multichannel-scattering character of a DWSL for $\eta=1$. The lowest quasienergy level (level 1) and the second-lowest one (level 2) supported by each site of the ponderomotive potential are indicated by the red and blue solid segments, respectively, and the given energy $E$ is represented by the green horizontal line. In addition, the ponderomotive potential is depicted for $\alpha=25$. (b) Schematic representation of the interchannel coupling between levels 1 and 2 belonging to the photon sidebands, $v$ and $\mu$, respectively, invoked by $V_{\mu \nu}(z)$. For more detail, consult the text.

component of it, $V_{\mu \mu}(z)$, is dominant, and the rest of the off-diagonal component, $V_{\mu \nu}(z)$ with $n \equiv \mu-v \neq 0$, has negligible contributions. With the increase of $\alpha$, on the whole, $V_{\mu \mu}(z)$ becomes weaker and its potential shape is made flatter, whereas $V_{\mu \nu}(z)$ with a larger $|n|$ becomes effective. For more detail, consult Figs. 2 and 3 of Ref. 19. A similar tendency also applies for $[1 / m]_{\mu \nu}(z)$, though its effect is considered weaker than that of $V_{\mu \nu}(z)$ because of the small difference of the masses in the barrier and well regions of the SL potential. $v_{\mu \nu}(z)$ depends on both of $F_{a c}$ and $\alpha$, not solely on $\alpha$, aside from $z$.

As shown below, Eq. (5) is regarded as the coupled equations for the multichannel scattering problem, where a scattering channel is designated by a photon index $v . \psi_{\nu}(z)$ is hereafter written as $\psi_{\nu \beta}(z)$ in order to specify the $\beta$ th solution of Eq. (5); the number of the independent solutions is as many as the number of open channels, represented as $N_{o}$. Figure 1(a) shows the schematic representation of the multichannel scattering character of the DWSL, where the allocation of the ponderomotive potentials, $\ldots, U_{v+1}(z), U_{v}(z), U_{v-1}(z), \ldots$, in the case of $\eta=1$ is indicated as functions of $z$. Here, $\eta=\Omega / \omega$ with $\Omega$ a Bloch frequency, and $U_{v}(z)$ is the ponderomotive potential for the $\nu$ th channel, defined as

$$
U_{v}(z)=F_{0} z+V_{\nu v}(z)+v_{\nu v}(z)+v \omega,
$$


in terms of the diagonal component of $V_{\mu \nu}(z)$. Further, the red and blue solid segments represent the lowest quasienergy level (termed as level 1) and the second-lowest quasienergy level (termed as level 2) supported by each site, respectively. For a given $E$ denoted by the green horizontal line, open boundary conditions should be imposed on $\left\{\psi_{\nu \beta}(z)\right\}$ at $z=z_{a s}<0$ with $\left|z_{a s}\right|=L_{S L} / 2+\alpha$, because $E>U_{v}\left(z_{a s}\right)$. Figure 1(b) shows the coupling scheme between level 1 (red) supported by $U_{v}(z)$ and level 2 (blue) supported by $U_{\mu}(z)$ with $\mu \neq v$, where this coupling is caused by $V_{\mu \nu}(z)$. In this figure, each level is broadened and further blurred (represented by gradation), because a quasienergy miniband formed by PAT would somewhat decay due to the dc-ZT effect mentioned in Sec. I. It should be noted that $V_{\mu \nu}(z)$ with $\mu \neq v$ vanishes at $z \leqslant z_{a s}$, and this fact ensures the existence of asymptotic states as open channels. Because of this property, the coupled equations of Eq. (5) that result from the Kramers-Henneberger transformation can be solved as multichannel scattering equations.

In the present framework, it is understood that both the PAT and the ac-ZT are induced by the off-diagonal component of $V_{\mu \nu}(z)$ between quasienergy levels supported by different ponderomotive potentials with $\mu \neq v$. To be precise, following the two-quasienergy-level scheme shown in Fig. 1(a), the PAT effect is generated by a transition between two quasienergetically degenerate level 1's (level 2's), where one level is supported by one site of $U_{\nu}(z)$ and the other level is supported by another adjacent site of $U_{\mu=v \pm \eta}(z)$ with $\eta=1$. In the case of an integer $\eta$ larger than unity, the ponderomotive interactions $V_{\mu \nu}(z)$ with $|\mu-v|=1, \ldots, \eta$ contribute to the PAT. Based on the perturbation expansion of $V_{\mu \nu}(z)$ with respect to $\alpha$, this effect is qualitatively understood as the $\eta$-photon sequential absorption/emission process. Similarly, the ac-ZT effect is caused by the transition mediated by $V_{\mu \nu}(z)$ between level 1 supported by $U_{\mu}(z)$ and level 2 supported by $U_{\nu}(z)$; the case of $|\mu-v|=1$ is most significant as long as $\alpha$ is not large. Accordingly, in the excess DOS picture of concern, both of the tunneling effects are treated on an equal footing in terms of the off-diagonal component of the ponderomotive interaction.

The scattering matrix represented as $S(E)$ is defined by the following incoming scattering-wave boundary condition for the open channel $v$ :

$$
\psi_{\nu \beta}\left(z_{a s}\right)=\chi_{\nu \beta}^{(+)}\left(z_{a s}\right)-\sum_{\beta^{\prime}=1}^{N_{o}} \chi_{\nu \beta^{\prime}}^{(-)}\left(z_{a s}\right) S_{\beta^{\prime} \beta}(E),
$$

where $\chi_{\nu \beta}^{( \pm)}(z)=\phi_{\nu}^{( \pm)}(z) \delta_{\nu \beta}$ and $\phi_{\nu}^{( \pm)}(z)$ is an energynormalized progressive wave in the direction of $\pm z$, satisfying the asymptotic field-free equation associated with an Airy function; here, the electron moves just under the potential field of $U_{v}^{(a s)}(z)=F_{0} z+V_{a s}+v \omega$ in place of $U_{v}(z)$ of Eq. (8). On the other hand, the boundary condition for the closed channel $v$ is given by

$$
\psi_{\nu \beta}\left(z_{a s}\right)=0 .
$$

The Floquet equation (5) can be solved by use of the $R$-matrix propagation technique with high numerical efficiency and accuracy. The $R$ matrix is defined as

$$
R_{\mu \nu}(z)=\sum_{\beta} \psi_{\mu \beta}(z)[b(z) \psi(z)]_{\beta \nu}^{-1},
$$

where $b_{\mu \nu}(z)$ is given as follows:

$$
\begin{aligned}
b_{\mu \nu}(z)= & \left\{\frac{1}{2 m[z+a(t)]}\right\}_{\mu \nu} \frac{\partial}{\partial z} \\
& +i\left\{\frac{F_{a s} \sin \omega t}{2 \omega}\left(\frac{1}{m[z+a(t)]}-\frac{1}{m_{a s}}\right)\right\}_{\mu \nu} .
\end{aligned}
$$

$S(E)$ is provided by matching the $R$ matrix thus calculated with a set of the boundary conditions of Eqs. (9) and (10) at $z=z_{a s} ;$ for more detail, consult Ref. 19 .

An excess DOS is defined by

$$
\rho^{(e x)}(E)=\frac{i}{2} \operatorname{Tr}\left\{[S(E)]^{-1} \frac{d S(E)}{d E}\right\}
$$

in terms of $S(E)$, and the lifetime of the concerned state with $E$ is given by

$$
\tau(E)=\frac{\rho^{(e x)}(E)}{N_{o}}
$$

(see Refs. 28-31). The excess DOS is also expressed as

$$
\rho^{(e x)}(E)=\rho(E)-\rho^{(a s)}(E),
$$

where $\rho(E)$ and $\rho^{(a s)}(E)$ represent the DOS of the concerned DWSL and that of a field-free asymptotic state corresponding to $\chi_{\nu \beta}^{( \pm)}(z)$, respectively. $\rho^{(a s)}(E)$ is given by

$$
\rho^{(a s)}(E)=\frac{\sqrt{2 m_{a s}}}{\pi F_{0}} \sum_{\beta=1}^{N_{o}} \sqrt{E-U_{\beta}\left(z_{a s}\right)},
$$

showing just structureless continuum; this expression is obtained by imposing the supplemental condition that $\chi_{\nu \beta}^{(-)}\left(z_{a s}\right)-\chi_{\nu \beta}^{(+)}\left(z_{a s}\right)=0$. Therefore, the electronic structure of the DWSL observed in $\rho^{(e x)}(E)$ is considered to be almost similar to that in $\rho(E)$, since $\rho^{(a s)}(E)$ is a slowly varying function of $E$ in the range of the first Brillouin zone with an interval of $\omega$. In addition, we have the periodicity relation

$$
\rho^{(e x)}(E)=\rho^{(e x)}(E+n \omega),
$$

with $n$ integer, because of

$$
S_{\beta^{\prime} \beta}(E)=S_{\beta^{\prime}+n, \beta+n}(E+n \omega) .
$$

\section{B. Excess density of states}

Equation. (13) will be rewritten in another form given by the sum of three contributions representing different physical mechanisms. Each physical mechanism plays a decisive role of analyzing the underlying physics of the excess DOS calculated on a basis of the framework of Sec. II A. First of all, it is noted that the scattering matrix can be read as

$$
S_{\beta^{\prime} \beta}(E)=e^{-i \delta_{\beta^{\prime}}(E)} \bar{S}_{\beta^{\prime} \beta}(E) e^{-i \delta_{\beta}(E)}
$$

in terms of the alternative scattering matrix $\bar{S}(E)$, which is defined in the following boundary condition:

$$
\psi_{\nu \beta}\left(z_{a s}\right)=\bar{\chi}_{\nu \beta}^{(+)}\left(z_{a s}\right)-\sum_{\beta^{\prime}=1}^{N_{o}} \bar{\chi}_{\nu \beta^{\prime}}^{(-)}\left(z_{a s}\right) \bar{S}_{\beta^{\prime} \beta}(E) .
$$

Here, $\bar{\chi}_{\nu \beta}^{( \pm)}(z)$ is a solution of the $v$ th single-channel equation

$$
\left[\bar{L}_{v \nu}(z)-E\right] \bar{\chi}_{\nu \beta}^{( \pm)}(z)=0
$$


and its asymptotic form is given as

$$
\bar{\chi}_{\nu \beta}^{( \pm)}\left(z_{a s}\right)=\chi_{\nu \beta}^{( \pm)}\left(z_{a s}\right) e^{ \pm i \delta_{\beta}(E)},
$$

where $\bar{L}_{v v}(z)$ is the diagonal part of $L_{\mu \nu}(z)$, that is,

$$
L_{\mu \nu}(z)=\delta_{\mu \nu} \bar{L}_{\nu v}(z)+\overline{\mathcal{H}}_{\mu \nu}(z)
$$

with $\overline{\mathcal{H}}_{\mu \nu}(z)=\left(1-\delta_{\mu \nu}\right) \mathcal{H}_{\mu \nu}(z)$. Actually, $\overline{\mathcal{H}}_{\mu \nu}(z) \approx V_{\mu \nu}(z)$ for $\mu \neq v$, since the off-diagonal contributions from the first and fourth terms of Eq. (2) are considered to be negligibly smaller than $V_{\mu \nu}(z)$. Therefore, the phase shift $\delta_{\beta}(E)$ is dominantly attributed to elastic scattering due to the ponderomotive potential $\bar{U}_{\beta}(z)$ defined as $\bar{U}_{\beta}(z)=U_{\beta}(z)-U_{\beta}^{(a s)}(z) \approx$ $V_{\beta \beta}(z)$.

According to Eq. (19), $\rho^{(e x)}(E)$ is recast into the form

$$
\rho^{(e x)}(E)=\rho_{0}^{(e x)}(E)+\bar{\rho}^{(e x)}(E),
$$

where

$$
\rho_{0}^{(e x)}(E)=\sum_{\beta} \frac{d \delta_{\beta}(E)}{d E}
$$

and

$$
\bar{\rho}^{(e x)}(E)=\frac{i}{2} \operatorname{Tr}\left\{[\bar{S}(E)]^{-1} \frac{d \bar{S}(E)}{d E}\right\} .
$$

Here, $\rho_{0}^{(e x)}(E)$ is expected to show peak spectra of shaperesonance levels supported by $U_{\beta}(z)$, the profile of which is represented by the well known Breit-Wigner formula with the Lorentzian profile with respect to $E$.

Further, the modified scattering matrix $\bar{S}(E)$ is rewritten as

$$
\bar{S}(E)=1-2 i \bar{T}(E)
$$

in terms of the corresponding transition matrix $\bar{T}(E)$. Putting Eq. (27) into Eq. (26) leads to the expression

$$
\bar{\rho}^{(e x)}(E)=\operatorname{Re} \sum_{\beta^{\prime} \beta}\left\{\left[\delta_{\beta^{\prime} \beta}+2 i \bar{T}_{\beta^{\prime} \beta}^{\dagger}(E)\right] \frac{d \bar{T}_{\beta \beta^{\prime}}(E)}{d E}\right\},
$$

where $\bar{\rho}^{(e x)}(E)=\left[\bar{\rho}^{(e x)}(E)\right]^{\dagger}$ is utilized, which is obtained from unitarity, $[\bar{S}(E)]^{\dagger} \bar{S}(E)=1$.

Actually, the modified transition matrix $\bar{T}(E)$ is given in terms of an interaction $\overline{\mathcal{V}}$ and a free Green function $\mathcal{G}(E)$ to be defined by the following Dyson equation:

$$
\bar{T}(E)=\overline{\mathcal{V}}+\overline{\mathcal{V}} \mathcal{G}(E) \bar{T}(E)=\overline{\mathcal{V}}+\bar{T}(E) \mathcal{G}(E) \overline{\mathcal{V}} .
$$

The second equality of the above equation is explicitly written as

$$
\bar{T}_{\tilde{\beta}_{1} \tilde{\beta}_{2}}=\overline{\mathcal{V}}_{\tilde{\beta}_{1} \tilde{\beta}_{2}}+\sum_{\tilde{\beta}_{i} \tilde{\beta}_{j}} \bar{T}_{\tilde{\beta}_{1} \tilde{\beta}_{i}} \mathcal{G}_{\tilde{\beta}_{i} \tilde{\beta}_{j}}(E) \overline{\mathcal{V}}_{\tilde{\beta}_{j} \tilde{\beta}_{2}},
$$

where $\overline{\mathcal{V}}$ and $\mathcal{G}$ are given as

$$
\overline{\mathcal{V}}_{\tilde{\beta}_{i} \tilde{\beta}_{j}}=\sum_{v_{i} v_{j}}\left\langle\bar{\chi}_{v_{i} \beta_{i}}^{(-)}\left|\overline{\mathcal{H}}_{v_{i} v_{j}}\right| \bar{\chi}_{v_{j} \beta_{j}}^{(-)}\right\rangle
$$

and

$$
\mathcal{G}_{\tilde{\beta}_{i} \tilde{\beta}_{j}}(E)=\sum_{v_{i} v_{j}}\left\langle\bar{\chi}_{v_{i} \beta_{i}}^{(-)}\left|G_{v_{i} v_{j}}\right| \bar{\chi}_{v_{j} \beta_{j}}^{(-)}\right\rangle
$$

with the Green function as

$$
G_{v_{i} v_{j}}(z)=\left[\frac{1}{E-\bar{L}(z)+i \epsilon}\right]_{v_{i} v_{i}} \delta_{v_{i} v_{j}},
$$

respectively. Here, the notation of $\tilde{\beta}_{i(j)}$ is introduced to represent a set of the indexes $\left(\beta_{i(j)}, E_{i(j)}\right) ; E_{i(j)}$ represents the $i(j)$ th quasienergy level associated with the $\beta_{i(j)}$ th singlechannel equation (21); the $E_{i(j)}$ dependence of $\bar{\chi}_{v_{i(j)} \beta_{i(j)}}^{(-)}$is omitted in Eqs. (31) and (32) for the sake of simplicity. $\epsilon$ is an infinitesimal positive number in Eq. (33). Further, in Eq. (30), it is understood that the quasienergy-on-shell condition, namely, the quasienergy conservation of $E=E_{1}=E_{2}$, is taken so that $\bar{T}_{\beta_{1} \beta_{2}}(E) \equiv \bar{T}_{\tilde{\beta}_{1} \tilde{\beta}_{2}}$.

According to Eq. (30), we obtain

$$
\begin{aligned}
\bar{T}_{\tilde{\beta}_{1} \tilde{\beta}_{2}} & =\overline{\mathcal{V}}_{\tilde{\beta}_{1} \tilde{\beta}_{2}}+\overline{\mathcal{T}}_{\tilde{\beta}_{1} \tilde{\beta}_{2}}+\sum_{\tilde{\beta}_{j}} \overline{\mathcal{T}}_{\tilde{\beta}_{1} \tilde{\beta}_{j}} \mathcal{G}_{\tilde{\beta}_{j} \tilde{\beta}_{j}}(E) \overline{\mathcal{V}}_{\tilde{\beta}_{j} \tilde{\beta}_{2}} \\
& =Z_{\tilde{\beta}_{1} \tilde{\beta}_{2}}^{\prime}+\sum_{\tilde{\beta}_{j} \tilde{\beta}_{j^{\prime}}} Z_{\tilde{\beta}_{1} \tilde{\beta}_{j}} \mathcal{G}_{\tilde{\beta}_{j} \tilde{\beta}_{j^{\prime}}}^{\prime}(E) Z_{\tilde{\beta}_{j^{\prime}} \tilde{\beta}_{2}}^{\prime},
\end{aligned}
$$

where

and

$$
\begin{gathered}
\overline{\mathcal{T}}_{\tilde{\beta}_{1} \tilde{\beta}_{2}}=Z_{\tilde{\beta}_{1} \tilde{\beta}_{2}}+\sum_{\tilde{\beta}_{j}} \overline{\mathcal{T}}_{\tilde{\beta}_{1} \tilde{\beta}_{j}} \mathcal{G}_{\tilde{\beta}_{j} \tilde{\beta}_{j}}(E) Z_{\tilde{\beta}_{j} \tilde{\beta}_{2}}, \\
Z_{\tilde{\beta}_{i} \tilde{\beta}_{j}}=\sum_{\tilde{\beta}_{j^{\prime}}} \overline{\mathcal{V}}_{\tilde{\beta}_{i} \tilde{\beta}_{j^{\prime}}} \mathcal{G}_{\tilde{\beta}_{j^{\prime}} \tilde{\beta}_{j^{\prime}}}(E) \overline{\mathcal{V}}_{\tilde{\beta}_{j^{\prime}} \tilde{\beta}_{j}}, \\
\mathcal{G}_{\tilde{\beta}_{j} \tilde{\beta}_{j^{\prime}}}^{\prime}(E)=\left(\frac{1}{[\mathcal{G}(E)]^{-1}-Z}\right)_{\tilde{\beta}_{j} \tilde{\beta}_{j^{\prime}}},
\end{gathered}
$$

$$
Z_{\tilde{\beta}_{1} \tilde{\beta}_{2}}^{\prime}=\overline{\mathcal{V}}_{\tilde{\beta}_{1} \tilde{\beta}_{2}}+Z_{\tilde{\beta}_{1} \tilde{\beta}_{2}} .
$$

Here, for a given complex value of $k$, one tackles an eigenvalue problem of the non-Hermitian matrix of $\mathcal{G}_{\tilde{\beta}_{j} \tilde{\beta}_{j^{\prime}}}^{\prime}(E)$ by setting $E=k^{2} / 2 m_{a s}$; the moduli of the resulting eigenvalues that are infinite correspond to poles of this Green function. In the case that $k$ is a complex number with a negative imaginary part, the associated eigenvector corresponds to a resonance state, where $E$ is located in the second Riemann sheet (unphysical sheet). ${ }^{42}$ We prefer to represent the existence of resonance poles in the Green function in an explicit form for the later discussion. To do this, $\mathcal{G}_{\tilde{\beta}_{j} \tilde{\beta}_{j^{\prime}}}^{\prime}(E)$ is rewritten as follows:

$$
\begin{aligned}
\mathcal{G}_{\tilde{\beta}_{j} \tilde{\beta}_{j^{\prime}}}^{\prime}(E)= & \mathcal{G}_{\tilde{\beta}_{j} \tilde{\beta}_{j}}^{\prime(0)}(E) \delta_{\tilde{\beta}_{j} \tilde{\beta}_{j^{\prime}}} \\
& +\mathcal{G}_{\tilde{\beta}_{j} \tilde{\beta}_{j}}(E) Z_{\tilde{\beta}_{j} \tilde{\beta}_{j^{\prime}}} \mathcal{G}_{\tilde{\beta}_{j^{\prime}} \tilde{\beta}_{j^{\prime}}}(E)\left(1-\delta_{\tilde{\beta}_{j} \tilde{\beta}_{j^{\prime}}}\right)+\cdots .
\end{aligned}
$$

where

$$
\begin{aligned}
\mathcal{G}_{\tilde{\beta}_{j} \tilde{\beta}_{j}}^{(0)}(E) & \equiv\left(\frac{1}{[\mathcal{G}(E)]^{-1}-Z_{\tilde{\beta}_{j} \tilde{\beta}_{j}}}\right)_{\tilde{\beta}_{j} \tilde{\beta}_{j}} \\
& =\frac{1}{E-E_{j}-Z_{\tilde{\beta}_{j} \tilde{\beta}_{j}}} .
\end{aligned}
$$

$\mathcal{G}_{\tilde{\beta}_{j} \tilde{\beta}_{j}}^{\prime(0)}(E)$ is obtained from the diagonal component of $\mathcal{G}^{\prime}(E)$ by making the ladder approximation with respect to the self-energy term $Z_{\tilde{\beta}_{j} \tilde{\beta}_{j}}$. It is seen that there are poles of 
$\mathcal{G}_{\tilde{\beta}_{j} \tilde{\beta}_{j}}^{\prime(0)}(E)$ at $E=\mathcal{E}_{\tilde{\beta}_{j}}-i \Gamma_{\tilde{\beta_{j}}} / 2$ in the complex $E$ plane with $\mathcal{E}_{\tilde{\beta_{j}}} \equiv E_{j}+\Delta E_{\tilde{\beta_{j}}}$. Here,

$$
\Delta E_{\tilde{\beta}_{j}}-i \Gamma_{\tilde{\beta}_{j}} / 2=Z_{\tilde{\beta}_{j} \tilde{\beta}_{j}},
$$

that is, $\Delta E_{\tilde{\beta}_{j}}$ and $\Gamma_{\tilde{\beta}_{j}} / 2$ correspond to the quasienergy shift to $E_{j}$ and the associated full width at half maximum, respectively.

By using Eq. (39), Eq. (34) is recast into

$$
\bar{T}_{\tilde{\beta}_{1} \tilde{\beta}_{2}}=W_{\tilde{\beta}_{1} \tilde{\beta}_{2}}+\sum_{\tilde{\beta}_{j}} \frac{Z_{\tilde{\beta}_{1} \tilde{\beta}_{j}} Z_{\tilde{\beta}_{j} \tilde{\beta}_{2}}^{\prime}}{E-\mathcal{E}_{\tilde{\beta}_{j}}+i \Gamma_{\tilde{\beta}_{j}} / 2},
$$

where

$$
\begin{aligned}
W_{\tilde{\beta}_{1} \tilde{\beta}_{2}}= & Z_{\tilde{\beta}_{1} \tilde{\beta}_{2}}^{\prime}+\sum_{\tilde{\beta}_{j} \tilde{\beta}_{j^{\prime}}\left(\neq \tilde{\beta}_{j}\right)} Z_{\tilde{\beta}_{1} \tilde{\beta}_{j}} \mathcal{G}_{\tilde{\beta}_{j} \tilde{\beta}_{j}}(E) \\
& \times Z_{\tilde{\beta}_{j} \tilde{\beta}_{j^{\prime}}} \mathcal{G}_{\tilde{\beta}_{j^{\prime}} \tilde{\beta}_{j^{\prime}}}(E) Z_{\tilde{\beta}_{j^{\prime}} \tilde{\beta}_{2}}^{\prime}+\cdots
\end{aligned}
$$

Further, putting Eq. (42) into Eq. (28) in view of $\bar{T}_{\beta_{1} \beta_{2}}(E)=$ $\bar{T}_{\tilde{\beta}_{1} \tilde{\beta}_{2}}$ and $\overline{\mathcal{V}}_{\tilde{\beta}_{1} \tilde{\beta}_{1}}=0, \bar{\rho}^{(e x)}(E)$ becomes

$$
\begin{aligned}
\bar{\rho}^{(e x)}(E) \equiv & \rho_{n r}^{(e x)}(E)+\rho_{r e s}^{(e x)}(E) \\
= & \sum_{\beta_{1}} \operatorname{Re}\left[D_{\beta_{1} \beta_{1}}+i C_{\beta_{1} \beta_{1}}\right. \\
& \left.+\sum_{\tilde{\beta_{j}}} \frac{B_{\beta_{1} \beta_{j}}+i A_{\beta_{1} \beta_{j}}}{E-\mathcal{E}_{\tilde{\beta_{j}}}+i \Gamma_{\tilde{\beta_{j}}} / 2}+\Delta_{\beta_{1}}(E)\right],
\end{aligned}
$$

where

$$
\rho_{n r}^{(e x)}(E)=\sum_{\beta_{1}} D_{\beta_{1} \beta_{1}}
$$

and

$$
\rho_{r e s}^{(e x)}(E) \approx \sum_{\beta_{1} \beta_{j} j} \frac{B_{\beta_{1} \beta_{j}}\left(E-\mathcal{E}_{\beta_{j}}\right)+A_{\beta_{1} \beta_{j}}\left(\Gamma_{\beta_{j}} / 2\right)}{\left(E-\mathcal{E}_{\tilde{\beta}_{j}}\right)^{2}+\left(\Gamma_{\tilde{\beta}_{j}} / 2\right)^{2}} .
$$

Here, $\Delta_{\beta_{1}}(E)$ in Eq. (44) represents the contribution from the terms including $\mathcal{G}_{\tilde{\beta}_{j} \tilde{\beta}_{j}}^{\prime(E)}(E)$ more than twice, and this is omitted in Eq. (46) just for the sake of simplicity.

Here, $A_{\beta_{1} \beta_{j}}, B_{\beta_{1} \beta_{j}}, C_{\beta_{1} \beta_{1}}$, and $D_{\beta_{1} \beta_{1}}$ are real. All of these values are regarded approximately as constant just in the vicinity of resonance at $E \approx \mathcal{E}_{\tilde{\beta}_{j}}$, and weakly dependent on $E$ off this resonance. All of these constants are defined as follows:

$$
\begin{aligned}
& B_{\beta_{1} \beta_{j}}+i A_{\beta_{1} \beta_{j}} \\
& =\frac{d\left(Z_{\tilde{\beta}_{1} \tilde{\beta}_{j}} Z_{\tilde{\beta}_{j} \tilde{\beta}_{1}}^{\prime}\right)}{d E}+2 i \sum_{\tilde{\beta}_{i}}\left[W_{\tilde{\beta}_{1} \tilde{\beta}_{i}}^{\dagger} \frac{d\left(Z_{\tilde{\beta}_{i} \tilde{\beta}_{j}} Z_{\tilde{\beta}_{j} \tilde{\beta}_{1}}^{\prime}\right)}{d E}\right. \\
& \left.\quad+Z_{\tilde{\beta}_{1} \tilde{\beta}_{j}}^{\prime \dagger} Z_{\tilde{\beta}_{j} \tilde{\beta}_{i}}^{\dagger} \frac{d W_{\tilde{\beta}_{i} \tilde{\beta}_{1}}}{d E} e^{i \Theta_{\tilde{\beta}_{j}}}\right],
\end{aligned}
$$

and

$$
D_{\beta_{1} \beta_{1}}+i C_{\beta_{1} \beta_{1}}=\frac{d W_{\tilde{\beta}_{1} \tilde{\beta}_{1}}}{d E}+2 i \sum_{\tilde{\beta}_{i}} W_{\tilde{\beta}_{1} \tilde{\beta}_{i}}^{\dagger} \frac{d W_{\tilde{\beta}_{i} \tilde{\beta}_{1}}}{d E},
$$

where

$$
\Theta_{\tilde{\beta}_{j}}=2 \tan ^{-1}\left[\frac{\Gamma_{\tilde{\beta}_{j}} / 2}{E-\mathcal{E}_{\tilde{\beta}_{j}}}\right] .
$$

As shown in Eq. (44), $\bar{\rho}^{(e x)}(E)$ consists of the nonresonant contribution $\rho_{n r}^{(e x)}(E)$ and the resonant contribution $\rho_{r e s}^{(e x)}(E)$. In $\rho_{\text {res }}^{(e x)}(E)$, the spectral shape is of a form akin to the Shore profile, ${ }^{43}$ indicating that the absorption lineshape is of Feshbach resonance; it is remarked that $\bar{\rho}^{(e x)}(E)$ is likely to be negative, and there is an additional contribution from $\Delta_{\beta_{1}}(E)$ [see right below Eq. (46)]. Hence, the parameters $A_{\beta_{1} \beta_{j}}$ and $B_{\beta_{1} \beta_{j}}$ play the roles of spectral intensity and spectral asymmetricity, respectively. When $E$ is close to the resonance quasienergy level, $\mathcal{E}_{\tilde{\beta}_{i}}$, according to Eq. (14), we have the phenomenological expression of the lifetime of the $\beta_{j}$ th resonance state as follows:

$$
\tau\left(\mathcal{E}_{\tilde{\beta}_{j}}\right) \approx\left(\frac{1}{N_{o}} \sum_{\beta_{1}} A_{\beta_{1} \beta_{j}}\right) \frac{2}{\Gamma_{\tilde{\beta}_{j}}},
$$

as long as $\left|\rho_{r e s}^{(e x)}\left(\mathcal{E}_{\tilde{\beta}_{j}}\right)\right| \gg\left|\rho_{0}^{(e x)}\left(\mathcal{E}_{\tilde{\beta}_{j}}\right)\right|,\left|\rho_{n r}^{(e x)}\left(\mathcal{E}_{\tilde{\beta}_{j}}\right)\right|$.

To summarize, the excess DOS of concern, $\rho^{(e x)}(E)$, is given by the sum of the single-channel resonance contribution of $\rho_{0}^{(e x)}(E)$, the multichannel nonresonance contribution of $\rho_{n r}^{(e x)}(E)$, and the multichannel resonance contribution of $\rho_{\text {res }}^{(e x)}(E)$. That is,

$$
\rho^{(e x)}(E)=\rho_{0}^{(e x)}(E)+\rho_{n r}^{(e x)}(E)+\rho_{r e s}^{(e x)}(E),
$$

where the respective terms are at least of zeroth, second, and third orders with respect to the off-diagonal component of $\overline{\mathcal{V}}$; see Eqs. (47) and (48). Thus, it is speculated that the higher-order contributions of $\rho_{n r}^{(e x)}(E)$ and $\rho_{r e s}^{(e x)}(E)$ begin to contribute to $\rho^{(e x)}(E)$ with the increase in $\alpha$. For very small $\alpha \approx 1, \rho^{(e x)}(E)$ is governed just by $\rho_{0}^{(e x)}(E)$, in which a single ponderomotive potential plays a decisive role of forming a shape-resonance peak. With increasing $\alpha$, the PAT becomes more important, leading to the quasienergyminiband formation. This effect would be effectively caused by $W_{\tilde{\beta}_{1} \tilde{\beta}_{1}}$ mediated by the ponderomotive interaction $\overline{\mathcal{V}}_{\tilde{\beta}_{1} \tilde{\beta}_{j}}$ with $\left|\beta_{1}-\beta_{j}\right|=1, \ldots, \eta$, as mentioned in Sec. II A. As $\alpha$ becomes further larger, $W_{\tilde{\beta}_{1} \tilde{\beta}_{1}}$ also causes the ac-ZT effect between different minibands of the original SLs, leading to the quasienergy-miniband deformation, as mentioned again in Sec. II A. Therefore, in these $\alpha$ regions, it is speculated that $\rho^{(e x)}(E)$ is governed by $\rho_{n r}^{(e x)}(E)$ as well as $\rho_{0}^{(e x)}(E)$.

With still further increase of $\alpha, \rho^{(e x)}(E)$ would be exclusively determined by $\rho_{r e s}^{(e x)}(E)$ with background contributions from $\rho_{n r}^{(e x)}(E)$. Thus, a great number of newly growing spectral peaks would manifest themselves around $E \approx \mathcal{E}_{\tilde{\beta}_{i}}$ due to the multichannel resonance scattering effect. From the mathematical point of view, such generation of many resonance states is considered to be due to the fact that the transcendental equation of $E=E_{j}+Z_{\tilde{\beta}_{j}, \tilde{\beta}_{j}}(E)$ with respect to $E$ likely has more than one solution in the complex $E$ plane when a ponderomotive interaction becomes strong to a certain extent. Here, this equation corresponds to the zeros of the denominator of Eq. (40), and the $E$ dependence of $Z_{\tilde{\beta}_{j}, \tilde{\beta}_{j}}$ of Eq. (36) is specified. If this ponderomotive interaction is not strong yet, just a single solution of $E \approx E_{j}+Z_{\tilde{\beta}_{j}, \tilde{\beta}_{j}}\left(E_{j}\right)$ is obtained from the above equation. In fact, it would be difficult to assign a certain spectral peak to either $\rho_{r e s}^{(e x)}(E)$ or $\rho_{n r}^{(e x)}(E)$ in a clear-cut way, and thus the border of $\alpha$ between the two mechanisms would not be well delimited. Hereafter, it is 
understood that the value of $\alpha$ at which $\rho^{(e x)}(E)$ starts to show the branching of a spectral peak into a couple of new resonance peaks is a rough border between $\rho_{r e s}^{(e x)}(E)$ and $\rho_{n r}^{(e x)}(E)$.

In addition, as $\alpha$ increases in this resonance region, the spectral width of $\rho^{(e x)}(E)$, namely $\Gamma_{\tilde{\beta_{j}}}$, tends to be more broadened, whereas the associated spectral peak does not always tend to be more enhanced. This is because $\tau\left(\mathcal{E}_{\tilde{\beta}_{j}}\right)$ given by Eq. (50) is determined by the product of $A_{\beta_{1} \beta_{j}}$ and $2 / \Gamma_{\tilde{\beta_{j}}}$, where $A_{\beta_{1} \beta_{j}}$ would be greater with increasing $\alpha$ against $2 / \Gamma_{\tilde{\beta_{j}}}$.

\section{RESULTS AND DISCUSSION}

The actual calculations are implemented for the SLs of $35 / 11 \mathrm{ML} \mathrm{GaAs} / \mathrm{Ga}_{0.75} \mathrm{Al}_{0.25} \mathrm{As}(1 \mathrm{ML}=2.83 \AA)$ with a lattice constant $d=246$ and barrier height of a confining quantumwell potential $V_{b}=7.8 \times 10^{-3}$. The material parameters employed here are the same as those in Ref. 19. Hereafter, the applied bias is set equal to $F_{0}=104.5 \mathrm{kV} / \mathrm{cm}$ which corresponds to the Bloch frequency of $\Omega \equiv F_{0} d=5 \times 10^{-3}$.

Figure 2 shows the quasienergy bands $E(\alpha)$ (abscissa) as a function of $\alpha$ (ordinate), which are calculated by use of the nearest-neighbor tight-binding model, incorporating the ac-ZT between WSL levels attributed to different SL-minibands. The ac-ZT is classified into the intra-WSL-site coupling and the inter-WSL-site coupling for the sake of convenience, as stated in Sec. I. Just the former coupling is included in the results of Fig. 2. The $E(\alpha)$ 's are calculated for $\eta=1$ with

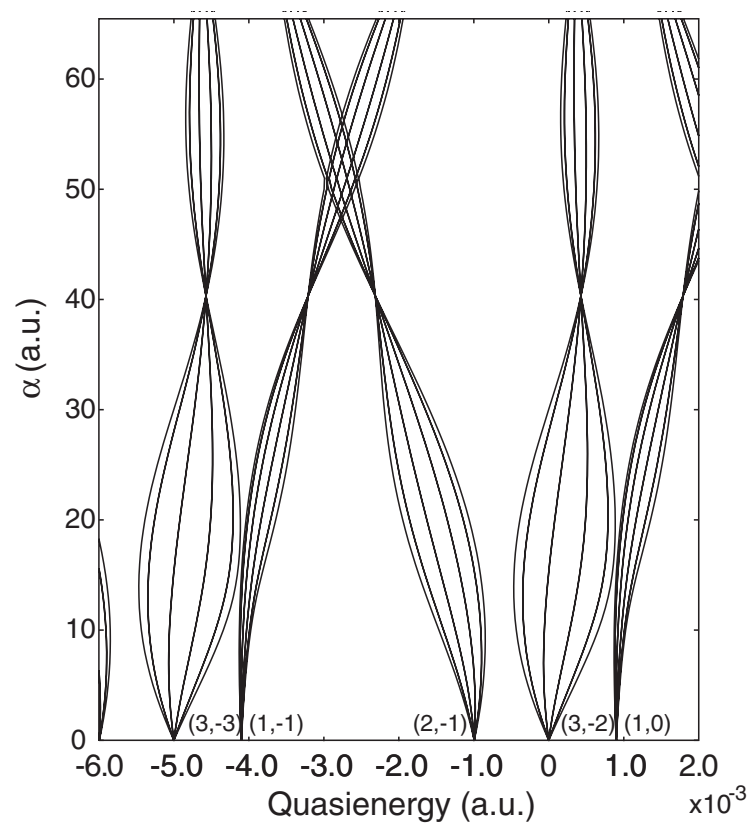

FIG. 2. The quasienergy bands $E(\alpha)$ (abscissa) as a function of $\alpha$ (ordinate) for $\eta=1$. The trace is the calculated result based on the tight-binding model with the lowest three SL minibands. Each lobe is labeled as $(b, j)$ with $b$ and $j$ as a SL-miniband index and a photon sideband index, respectively. The different lines within each lobe correspond to the quasienergy values of different $|k|$ 's, where $k$ represents the Bloch momentum of the original SLs. It is noted that dynamic localization occurs at $\alpha=40.35$. For more detail, consult the text. the energetically lowest three SL minibands. Hereafter, the SL-miniband index is labeled as $b$. In the present SLs, the energetically lowest two minibands with $b=1$ and 2 are attributed to discrete quantum-well levels, the energy levels of which are less than $V_{b}$, whereas the third energetically lowest miniband and higher with $b \geqslant 3$ are attributed to continuum quantum-well levels, the energy levels of which are larger than $V_{b}$.

In Fig. 2, each gourd-shaped lobe is labeled as $(b, j)$ with $j$ a photon sideband index. This label is considered to be a good quantum number just for small $\alpha$ 's; the Bloch momentum of the original SLs, $k$, remains a good quantum number even in the whole $\alpha$ region. In addition, it is noted that dynamic localization occurs at $\alpha=40.35$. It is considered that the calculated result is numerically convergent almost in the whole $\alpha$ region concerned here. The grounds for this are described in the Appendix in detail, since the justification of this convergence seems substantial for the comparison with the result obtained based on the excess-DOS picture. In the subsequent discussion we employ the $E(\alpha)$ of concern as the reference of comparisonwith the results of the multichannel scattering calculations described in Sec. II A.

It is expected that the alteration of excess DOS, $\rho^{(e x)}(E)$, with respect to $\alpha$ can be understood based on Eq. (51). Figure 3 shows the calculated results of $\rho^{(e x)}(E)$ as a function of $E$ for $\eta=1$, which are depicted by red solid lines. In addition, $E(\alpha)$ of Fig. 2 is also included for the sake of comparison. In Fig. 3(a), $\rho^{(e x)}(E)$ 's for $\alpha=1$ and 25 are shown in the $E$ region where the quasienergy band $(1,-1)$ is dominant; because of Eq. (17), the profiles of $\rho^{(e x)}(E)$ 's are the same as those in the $E$ region where the parent band $(1,0)$ is dominant. The single sharp peak and the concave spectra with a double peak are discerned at $\alpha=1$ and 25, respectively.

The peak observed at $\alpha=1$ is attributed to the energetically lowest WSL level for $\alpha=0$ assigned to the miniband index of $b=1$ and the WSL index of $n_{\mathrm{WSL}}=-1$. The relatively longhowever, finite-lifetime of this level implies the significance of the continuum effect due to the dc-ZT, though following the quasienergy picture, the associated DOS just becomes infinite. Such behavior is well understood by the shape resonance caused by the single-channel scattering in terms of $\rho_{0}^{(e x)}(E)$ [see Eq. (25)]. The concaved profile observed at $\alpha=25$ is reminiscent of the DOS of a DWSL with $E(\alpha) \propto \cos (k d)$ where $k$ represents the Bloch momentum. Here, the prominent double peak just corresponds to the van Hove singularities, and the finiteness of these peaks again suggests the continuum effect. It should be noted that excess-DOS picture appears to conform well with the quasienergy picture as long as both of the intersite ac-ZT and the dc-ZT have little effect on the excess DOS in the small- $\alpha$ region.

Both the PAT and ac-ZT, resulting in quasienergy-band formation and deformation, respectively, are described by $\rho_{n r}^{(e x)}(E)$, as mentioned in Sec. II B [see Eq. (45)]. In Fig. 3(a), two reference spectra, depicted by the black and blue dashed lines, are also shown for $\alpha=25$. Here, the former spectra are calculated by excluding all off-diagonal components of ponderomotive interaction $V_{\mu \nu(\neq \mu)}$, and thus these spectra form just a single peak attributed to $\rho_{0}^{(e x)}(E)$. The latter spectra are obtained by including the components with $|\mu-\nu|=1(=\eta)$, 

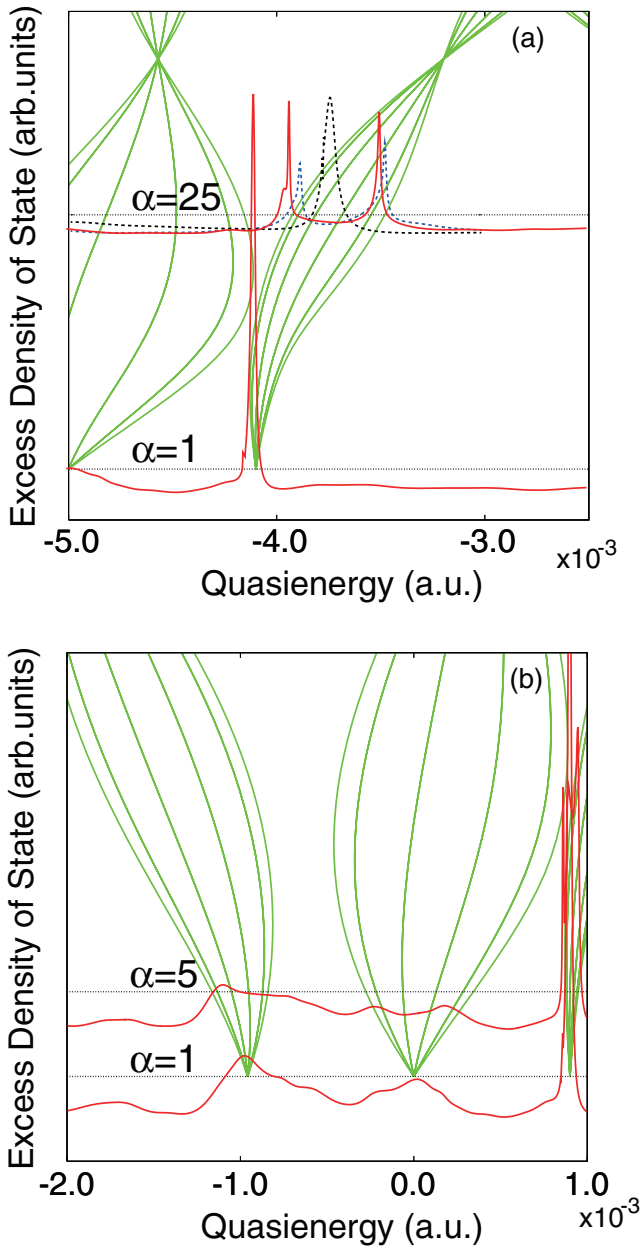

FIG. 3. (Color online) The excess DOS, $\rho^{(e x)}(E)$, as a function of quasienergy, $E$, for $\eta=1$, where $E(\alpha)$ of Fig. 2 is also included for the sake of comparison. (a) $\rho^{(e x)}(E)$ for $\alpha=1$ and 25 (depicted by the red solid lines) in the $E$ region where the quasienergy band $(1,-1)$ is dominant. In addition, two reference spectra are included for $\alpha=25$, which are depicted by the black and blue dashed lines. For more detail, consult the text. (b) $\rho^{(e x)}(E)$ for $\alpha=1$ and 5 (depicted by the red solid lines) in the $E$ region where the quasienergy bands $(2,-1)$ and $(3,-2)$ are dominant.

and a concave profile with a double peak is observed, as suggested in Sec. II. This is almost in accord with the profile of the spectra depicted by the red solid line, where these spectra are obtained by a full calculation including all of the off-diagonal components. The comparison among these three spectra really demonstrates the significant role of $V_{\mu \nu(\neq \mu)}$ with $|\mu-v|=1(=\eta)$ in forming the PAT and ac-ZT due to $\rho_{n r}^{(e x)}(E)$; the reason for excluding the contribution of $\rho_{r e s}^{(e x)}(E)$ from this discussion is described later.

On the other hand, in Fig. 3(b), $\rho^{(e x)}(E)$ 's for $\alpha=1$ and 5 are shown in the $E$ region where the quasienergy bands $(2,-1)$ and $(3,-2)$ are dominant. The spectral peaks look blurred due to the relatively strong effect of dc-ZT. Therefore, the applicability of the quasienergy picture seems limited in the DWSL states with $b \geqslant 2$.

Figure 4 shows the alteration of $\rho^{(e x)}(E)$ in the whole $\alpha$ region concerned here for $\eta=1$, where $E(\alpha)$ of Fig. 2 is also

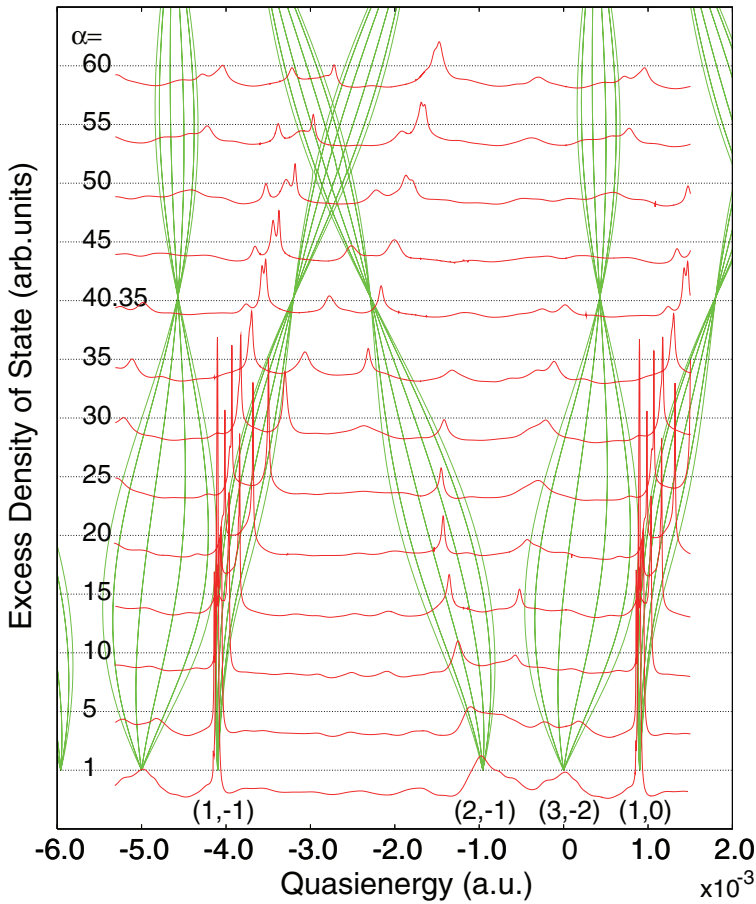

FIG. 4. (Color online) The excess DOS, $\rho^{(e x)}(E)$, as a function of quasienergy, $E$, in the extended $\alpha$ region for $\eta=1$. $E(\alpha)$ of Fig. 2 is also included for the sake of comparison. It is noted that dynamic localization occurs at $\alpha=40.35$.

included for the sake of comparison. Further, in Fig. 5, the alteration of $\rho^{(e x)}(E)$ for $\eta=3$ is shown for the purpose of comparison with the results of Fig. 4, where the associated

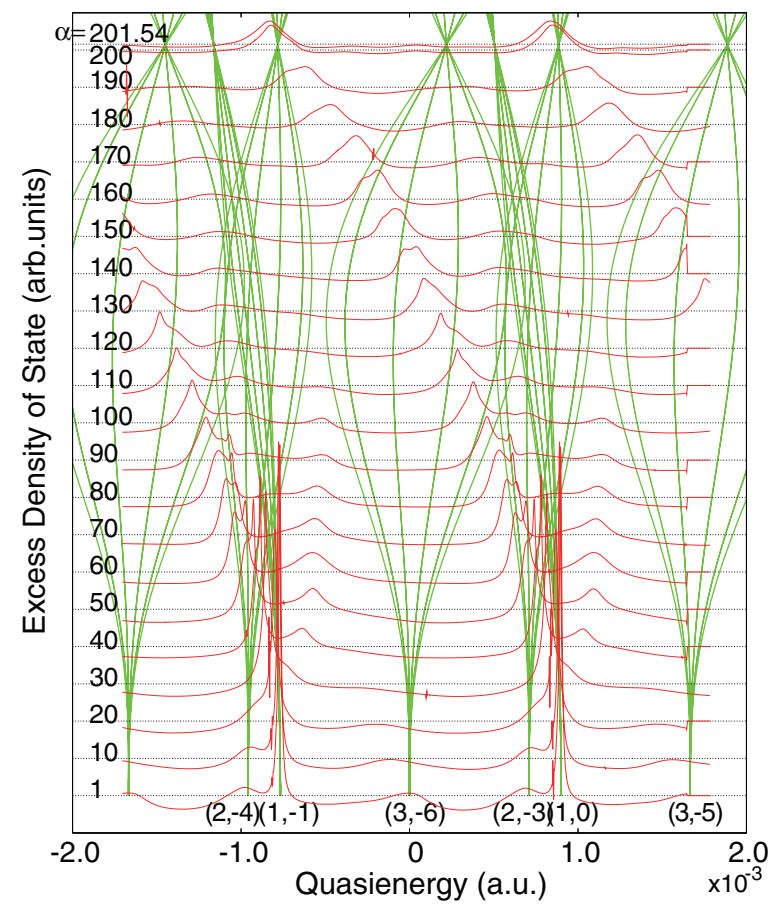

FIG. 5. (Color online) The same as Fig. 4 but $\eta=3$. $E(\alpha)$ relevant to this case is also included for the sake of comparison. It is noted that dynamic localization occurs at $\alpha=201.54$. 
$E(\alpha)$ is also included. It is speculated that this quasienergy band is numerically more convergent than that of Fig. 2 for the following reason. As stated in the Appendix, the ac-ZT coupling is proportional to $F_{a c}$, and there is the relation of $F_{a c} \propto \alpha / \eta^{2}$ obtained from Eq. (3). Accordingly, for example, $F_{a c}$ at $\alpha=180$ for $\eta=3$ equals that at $\alpha=20$ for $\eta=1$, where it is noted that the convergence of the results of Fig. 2 is already confirmed in the Appendix. Therefore, it is certain that the results of $E(\alpha)$ included in Fig. 5 converge well in the whole $\alpha$ region concerned here.

With the increase in $\alpha$, it is seen that the peak positions of $\rho^{(e x)}(E)$ for $\eta=1$ are more deviated from those provided by $E(\alpha)$. Moreover, the van Hove structure is no longer observed even in the photon sideband $(1, j)$, differing from the spectra shown in Fig. 3(a). The same result also holds correctly in the case of $\eta=3$. This implies that the quasienergy picture does not hold in the large- $\alpha$ region because of the growing importance of the continuum effect due to the intersite coupling.

Figure 6 shows the variance of main-peak positions of $\rho^{(e x)}(E)$ for $\eta=1$ in the $E-\alpha$ plane as a supplement of Fig. 4. The sequences of peaks labeled by the pair $\mathrm{A}$ and $\mathrm{A}^{\prime}$ show the alteration of $\rho^{(e x)}(E)$ originally pertaining to the quasienergy band $(1,-1)$. Similarly, the sequences of peaks labeled by the pair $\mathrm{B}$ and $\mathrm{B}^{\prime}$ and the pair $\mathrm{C}$ and $\mathrm{C}^{\prime}$ correspond to the quasienergy bands $(2,-1)$ and $(3,-2)$, respectively. These pairs of peaks would be considered vestiges of formation of PAT and ac-ZT. The sequences $\mathrm{B}^{\prime}$ and $\mathrm{C}$ are merged into the sequence labeled by $\mathrm{D}$. The other sequences labeled by $\mathrm{a}$,

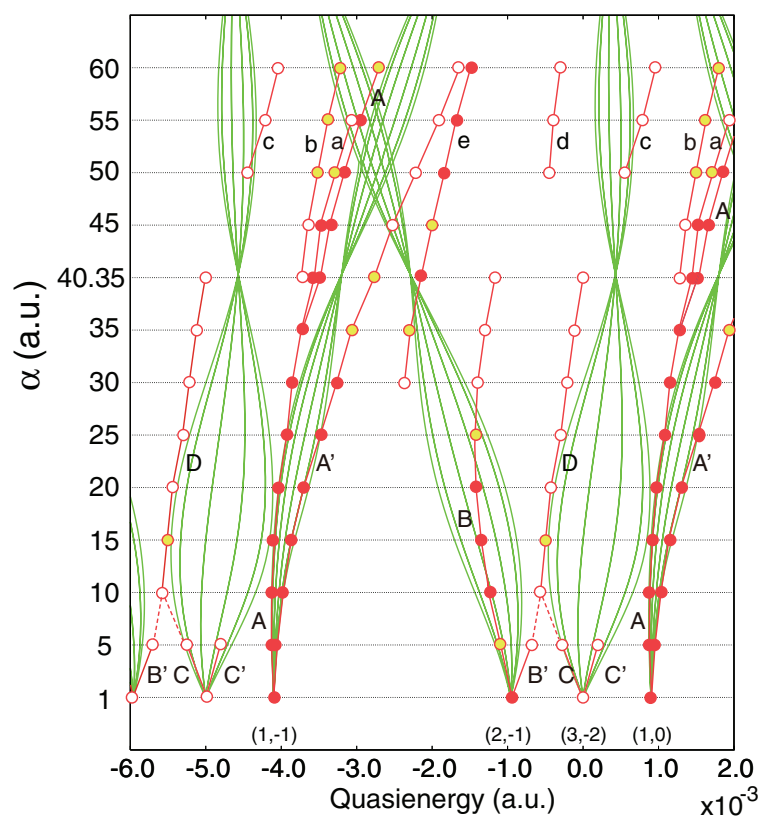

FIG. 6. (Color online) The variance of main-peak positions of $\rho^{(e x)}(E)$ in the $E-\alpha$ plane for $\eta=1$. Filled circles with red and yellow represent the peaks with $\rho^{(e x)}(E)>0$ and $\rho^{(e x)}(E) \approx 0$, respectively, while open circles represent the peaks with $\rho^{(e x)}(E)<0$. The sequences of peaks are connected by dotted lines to aid the presentation, and they are labeled as $\mathrm{A}^{(\prime)}, \mathrm{B}^{(\prime)}, \mathrm{C}^{(\prime)}, \mathrm{D}^{(\prime)}, \mathrm{a}, \mathrm{b}, \mathrm{c}, \mathrm{d}$, and e. $E(\alpha)$ of Fig. 2 is also included for the sake of comparison.
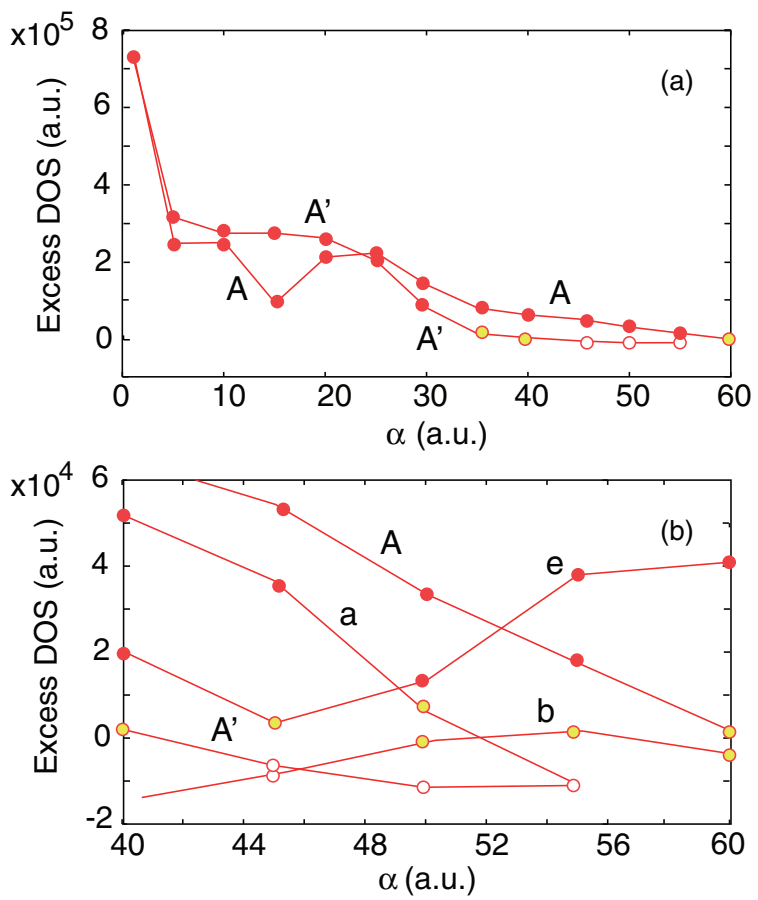

FIG. 7. (Color online) (a) The variance of main-peak values of $\rho^{(e x)}(E)$ as a function of $\alpha$ for $\eta=1$. The sequences of peaks are connected by solid lines to aid the presentation. (b) The same as panel (a) but in the limited- $\alpha$ region. The meanings of red and yellow filled circles, of open circles, and of the labels $\mathrm{A}, \mathrm{A}^{(\prime)}, \mathrm{a}, \mathrm{b}$, and e are the same as Fig. 6.

$\mathrm{b}, \mathrm{c}, \mathrm{d}$, and e are relevant to the peaks appearing only when $\alpha>35$. In particular, the primary sequence of A branches out into the sequences a and $\mathrm{b}$. It is obvious that such branching is attributed to neither PAT nor ac-ZT. Further, Fig. 7 shows the variance of main-peak values of $\rho^{(e x)}(E)$ as a function of $\alpha$ for $\eta=1$ as a supplement of Fig. 4. As regards lifetime, see Eq. (14), where $N_{0}=21$ in the present calculations, and note that 1 a.u. $=2.419 \times 10^{-17} \mathrm{~s}$; for example, the lifetime of $\mathrm{A}^{\prime}$ at $\alpha=15$ is almost equal to 0.34 ps. As seen in Fig. 7(a), the peak values of $\mathrm{A}$ and $\mathrm{A}^{\prime}$ for $\alpha \leqslant 25$ look similar to each other aside from those at $\alpha=15$; the cause of this exception is not definite at present. It is seen in Fig. 7(b) that resonance peaks of $a, b$, and e grow up, and then diminish in the region of $\alpha \geqslant 40$,

Now, discussion is directed toward clarifying the difference of the pattern of change in $\rho^{(e x)}(E)$ for $\eta=1$ from that for $\eta=3$. As shown in Figs. 4 and 6 , for $\alpha$ 's greater than 35 , obviously a great number of the newly growing peaks manifest themselves. In contrast, in Fig. 5, the spectra indicate monotonic change with pronounced redshift of the single peak attributed mostly to the photon sideband $(1, j)$ in the whole $\alpha$ region. Such behavior of $\rho^{(e x)}(E)$ would be understood in the light of Eq. (51). For $\eta=1$, it is speculated that $\rho^{(e x)}(E)$ is governed by $\rho_{\text {res }}^{(e x)}(E)$ in the region of $\alpha>35$, since the spectral pattern mentioned above is discriminated from that observed in the region of $\alpha<35$ where $\rho_{n r}^{(e x)}(E)$ as well as $\rho_{0}^{(e x)}(E)$ is dominant; this is the reason why $\rho_{\text {res }}^{(e x)}(E)$ is excluded from the discussion of Fig. 3(a). Hence, many of the peaks arise from multichannel resonance scattering 
mediated by the ponderomotive interactions. Moreover, it is observed that, for greater $\alpha$, the spectral width of $\rho^{(e x)}(E)$ tends to be more broadened, while the associated spectral height does not always tend to be more enhanced. Such a tendency would be in conformity with the expected effect of $\rho_{\text {res }}^{(e x)}(E)$ mentioned at the end of Sec. II B. In addition, dynamic localization expected at $\alpha=40.35$ by the quasienergy picture no longer persists in Fig. 4; such a disappearance of dynamic localization is understood by the intrinsic instability due to dynamic Fano resonance. ${ }^{19}$ Incidentally, although the manifestation of such complicated spectral structure for $\eta=1$ was already mentioned in Ref. 19, the analysis of its cause did not seem as evident as that made above in the present study.

On the other hand, for $\eta=3$, it is considered that $\rho^{(e x)}(E)$ is still dominated by $\rho_{n r}^{(e x)}(E)$ and $\rho_{0}^{(e x)}(E)$ in the $\alpha$ region concerned here, since there is no sign characteristic of multichannel resonance due to $\rho_{r e s}^{(e x)}(E)$. To be precise, the redshift shown in $\rho^{(e x)}(E)$ for $\eta=3$ is attributed mostly to $\rho_{n r}^{(e x)}(E)$. This is confirmed by comparing these spectra of Fig. 5 with those obtained by excluding all of the off-diagonal components of the ponderomotive interaction; the reference spectra thus obtained correspond just to $\rho_{0}^{(e x)}(E)$, showing a slight blueshift of spectral peak, not redshift, with the increase of $\alpha$ (though not shown here). Actually, the coupling $\mathcal{V}_{\tilde{\beta}_{i} \tilde{\beta}_{j}}$ with $\beta_{i} \neq \beta_{j}$ is considered relatively small in the whole region of $\alpha$. This is because, differing from the case of $\eta=1$, it is not likely that at the same time both of the single-channel wave functions, $\bar{\chi}_{\nu \beta_{i}}^{(-)}(z)$ and $\bar{\chi}_{v \beta_{j}}^{(-)}(z)$, will have locally dominant contributions due to shape resonance at the quasienergies, $E_{i}$ and $E_{j}$, respectively [for the definitions of $\bar{\chi}_{\nu \beta_{i}}^{(-)}(z)$ and $\mathcal{V}_{\tilde{\beta}_{i} \tilde{\beta_{j}}}$, see Eqs. (22) and (31), respectively]. We note that, apparently, the effect of the ponderomotive interaction $V_{\mu \nu}$ with $n=\mu-v \neq 0$ on the spectra at the value of $\alpha=200$ for $\eta=3$ would be more substantial than the spectra at the value of $\alpha=60$ for $\eta=1$, since this interaction depends just on $\alpha$ aside from $z$, as stated in Sec. II. Nevertheless, higher-order ponderomotive interactions with large values of $|n|$ are not particularly important in the case of $\eta=3$ for the above-mentioned reason. In addition, $\rho^{(e x)}(E)$ at $\alpha=201.54$ does not show any characteristic spectra, though dynamic localization is expected at this value, following the quasienergy picture. It is considered that sharp spectra predicted in dynamic localization ${ }^{44}$ are reduced and broadened by both effects of dc-ZT and ac-ZT, rather than by dynamic Fano resonance suitable for the case of $\eta=1$ mentioned above.

Incidentally, it is worth mentioning the possibility of an alternative mechanism for tunneling ionization. ${ }^{45}$ This mechanism is triggered by an electron tunneling into the continuum from a static tilted SL-confining potential, followed by the acceleration of this electron by an intense laser field; this differs from the mechanism discussed thus far, which follows the multiphoton process. These two mechanisms are distinguished by what is called the Keldysh parameter. This dimensionless parameter $\gamma$ is defined as

$$
\gamma=\sqrt{\frac{V_{b}}{2 U_{p}}},
$$

with the ponderomotive energy $U_{p}$ equal to $F_{a s}^{2} / 4 m_{b} \omega^{2}$. The multiphoton process is believed to be dominant in the region of $\gamma>1$, while tunneling ionization is possibly dominant in the region of $\gamma<1$. We obtain $\gamma=1.5$ for $\alpha=60$ in Fig. 4 , and $\gamma=1.35$ for $\alpha=200$ in Fig. 5. Thus, the whole $\alpha$ regions concerned in Figs. 4 and 5 are found to belong to $\gamma>1$. Therefore, it is convincing that the obtained results shown in these figures are safely understood in terms of Eq. (51) for the multiphoton process, and the possibility of tunneling ionization is left out ofthe discussion.

The multichannel resonance mentioned here is usually understood based on the Fano decay mechanism. Actually, in Ref. 19, the instability of dynamic localization is attributed to this mechanism, where two dynamic-localization states pertaining to the photon sidebands, $(1, j)$ and $\left(b^{\prime}, j^{\prime}\right)$ with $b^{\prime} \geqslant 2$, are coupled to each other; the former state is regarded as an almost discrete state, while the latter state is regarded as a continuum state since it is broadened to a certain extent due to the dc-ZT effect. In the present case, the $\mu$ th and $v$ th photon sidebands are already broadened due to PAT, and further, they are blurred to a certain extent by the shape resonance attributed to the potentials of $U_{\mu}(z)$ and $U_{v}(z)$, as shown in Fig. 1(b). If the condition of this figure is met that the $v$ th state is relatively narrow, and the $\mu$ th one is much broadened, the Fano-like decay would be effectively caused, similarly to the above case of dynamic localization. Strictly speaking, such a decay mechanism would differ from the original Fano decay mechanism. In the former, the $v$ th structured-continuum state decays into the $\mu$ th structured-continuum state; that is, one structured-continuum state transitions into another structured-continuum state. On the other hand, in the latter, a pure discrete state decays into an unstructured-continuum state; that is, a discrete state transitions into an unstructuredcontinuum state. ${ }^{46}$ Thus, to avoid unnecessary confusion regarding terminologies, the present case is referred to as "Fano-like" resonance instead of Fano resonance.

Finally, we mention the pattern of change in the spectra with $\eta=2$, where the calculated results are shown in Fig. 4 of Ref. 19 (not shown here again) with $\gamma=1.80$ for the maximum value of $\alpha=100$. It is seen in the concerned spectra that the two representative features found in the spectra with $\eta=1$ and $\eta=3$ coexist. That is, in the large- $\alpha$ region, the spectral peaks attributed to the photon sideband $(1, j)$ show a pronounced redshift, similarly to the case of $\eta=3$; and at the same time the spectra accompany a couple of the newly growing peaks, similarly to the case of $\eta=1$. Therefore, this case is considered to be intermediate between the cases of $\eta=1$ and 3 .

\section{CONCLUSION}

We demonstrate the significance of the multichannel continuum effects on the DWSL on the basis of the excess-DOS picture. The excess DOS, $\rho^{(e x)}(E)$, is shown to have distinct contributions from $\rho_{0}^{(e x)}(E), \rho_{n r}^{(e x)}(E)$, and $\rho_{r e s}^{(e x)}(E)$. With the increase in $\alpha$, the importance of the interchannel ponderomotive interactions increases. For very small $\alpha \approx 1, \rho^{(e x)}(E)$ shows just the single-peak spectra broadened somewhat due to the shape-resonance effect arising from a ponderomotive potential, namely, a diagonal component of the ponderomotive 
interaction. Here $\rho^{(e x)}(E)$ is exclusively governed by $\rho_{0}^{(e x)}(E)$. With increasing $\alpha$, the interchannel ponderomotive interaction comes into play, giving rise to the quasienergy-band formation and deformation due to the PAT and ac-ZT, respectively, though the observed spectra still look uncomplicated. Here $\rho^{(e x)}(E)$ is governed by $\rho_{n r}^{(e x)}(E)$ rather than $\rho_{0}^{(e x)}(E)$. With still further increase of $\alpha$, a great number of newly growing spectral peaks manifest themselves due to the Fano-like resonance mechanism caused by the multichannel ponderomotive interaction. Here $\rho^{(e x)}(E)$ is governed by $\rho_{\text {res }}^{(e x)}(E)$. We conclude that the excess DOS is considered to be a unified tool for exploring electronic properties of a DWSL in the whole $\alpha$ region; in particular, in the relatively small- $\alpha$ region, we illustrate that the excess DOS successfully reproduces the van Hove singularities characteristic of quasienergy minibands, which are conventionally evaluated by means of the tight-binding approximation without the continuum effects.

As regards the property of the DWSL in the still larger- $\alpha$ region than that presented in this study, chaotic scattering is expected to manifest itself, since classical motion of the electron in the DWSL is approximately considered to be the analog of a kicked rotator, and thus this is understood by means of the Hénon map. ${ }^{47}$ Actually, it is shown in Ref. 18 that time delay obtained from this classical motion of the electron forms a fractal structure equivalent to chaos. The excess-DOS picture developed here would represent the associated quantum-chaos of the DWSL as the manifestation of a great number of multichannel resonance states in such a fractal manner; as far as we know, this is thus far described based on the random matrix theory. ${ }^{18}$ In this context, the complication of the excess-DOS spectra observed in this study might be considered as a transient phenomenon from the regular region to the chaotic region, as long as the Keldysh parameter $\gamma$ is larger than unity.

Next, brief mention is made of the importance of the present study in the new research field of strong excitation of semiconductors. ${ }^{3}$ This field has been opened up by the recent exploration of intense terahertz waves; for example, destabilization of excitons dressed by a terahertz wave with peak electric field of the order of $100 \mathrm{kV} / \mathrm{cm}$ and higher is observed in semiconductor multiple quantum wells. ${ }^{48}$ The physical mechanisms causing the destabilization of electronic states are broadly classified into a multiphoton process, a tunneling ionization process, and an avalanche ionization process in terms of applied electric-field strength and frequency. ${ }^{19}$ There seems to be no theoretical model that can cross over all of these processes in a unified manner. The $R$-matrix Floquet theory relevant to the multiphoton process could be one of the promising frameworks that can describe the tunneling ionization process to a certain extent in the region of $\gamma<1$, though it is challenging and demanding. Moreover, the DWSL concerned here is also regarded as important, because this is the primitive system that provides the initial characterization of destabilization mechanisms of more complicated systems due to intense laser irradiation.

Finally, the present status of investigations of the manybody effect on the DWSL is mentioned; this effect is assumed to be neglected from the beginning in this paper. Although a number of studies of the DWSL have been focused on its importance ${ }^{49-53}$ these studies are limited to a small $\alpha$ region, and thus the significance of the ac-ZT interaction is overlooked to the best of our knowledge. Recently, two of the authors have demonstrated the manifestation of an asymmetric Fano lineshape of a photodressed-exciton state in interband absorption spectra, ${ }^{40,41}$ though this study just addresses the few-body problem. Further study is in progress, taking account of the many-body effect within the mean-field approximation based on the semiconductor-Bloch equations. ${ }^{54}$

\section{ACKNOWLEDGMENTS}

We are grateful to the reviewers of this paper for detailed and crucial comments. This work was supported by Grants-in-Aids for Scientific Research on Innovative Areas "Optical science of dynamically correlated electrons (DYCE)" (Grant No. 21104504) of the Ministry of Education, Culture, Sports, Science and Technology (MEXT), Japan. Numerical calculations were carried out on the T2K-Tsukuba System in the Center for Computational Sciences at the University of Tsukuba.

\section{APPENDIX: NUMERICAL ACCURACY OF CALCULATED QUASIENERGY BANDS}

The numerical accuracy of the quasienergy-band $E(\alpha)$ obtained by the lowest-three miniband calculation shown in Fig. 2 is confirmed by a model calculation including a higher miniband contribution. Figure 8 shows the allocation of quasienergy levels of $(b, j)$ 's at $\alpha \rightarrow 0$ in the interval of $E=$ $-6 \times 10^{-3}$ to $2 \times 10^{-3}$. Here, these levels are represented by $\epsilon_{(b, j)}$ 's. It is noted that the $\epsilon_{(b, j)}$ 's are estimated approximately from Fig. 1 of Ref. 27; the quasienergy difference of $\epsilon_{(b \geqslant 2,0)}$ from $\epsilon_{(1,0)}$ is approximately $3 \omega, 9 \omega, 18 \omega, 30 \omega$, and $48 \omega$ for $b=2,3,4,5$, and 6 , respectively, with $\omega=5 \times 10^{-3}$, since higher accuracy is unnecessary, as noted in the following discussion. Here, the ac-Zener coupling between the levels of $\epsilon_{(b, j)}$ and $\epsilon_{\left(b^{\prime}, j^{\prime}\right)}$ is represented as $X_{(b, j),\left(b^{\prime}, j^{\prime}\right)}$. This is considered

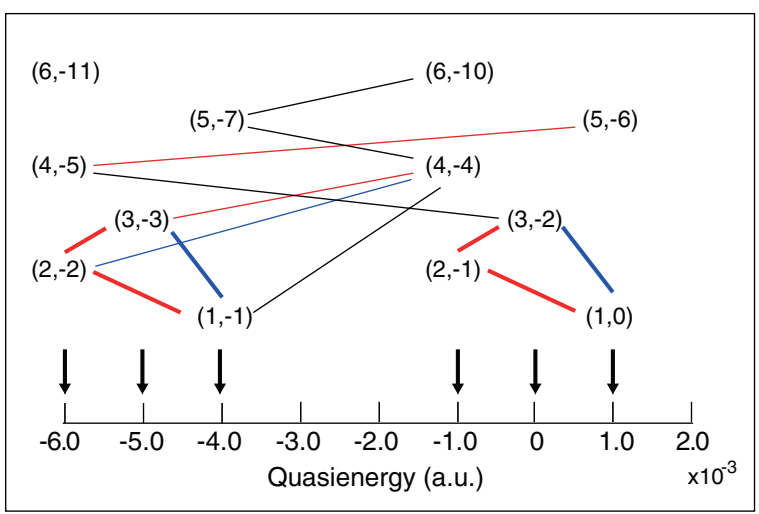

FIG. 8. (Color online) The schematic allocation of quasienergy levels, $\epsilon_{(b, j)}$ 's, at $\alpha \rightarrow 0$ in the interval of $E=-6 \times 10^{-3}$ to $2 \times 10^{-3}$. The positions of $(b, j)$ 's for $b=1-6$ are shown by downward allows. A pair of $(b, j)$ 's strongly coupled by the ac-Zener effect is connected by a solid line, where the red, blue, and black lines show that the associated transition is dominated by the first-, second-, and third-order couplings, respectively. The thick solid lines stand for the couplings included in the lowest-three SL-miniband calculations of Fig. 2. For more detail, consult the text. 
TABLE I. The eigenvalues (in units of a.u.), $\mathcal{E}_{(b, j)}^{(\mathrm{I} / \mathrm{II})}$, s, obtained by employing Base I and Base II. For the meaning of Base I and Base II, consult the text. Here, $\Delta \mathcal{E}_{(b, j)} \equiv \mathcal{E}_{(b, j)}^{(\mathrm{II})}-\mathcal{E}_{(b, j)}^{(\mathrm{I})} \cdot \mathcal{E}_{(2,-1)}^{(\mathrm{I} / \mathrm{II})}$ and $\mathcal{E}_{(3,-2)}^{(\mathrm{I} / \mathrm{II})}$ are provided by $\mathcal{E}_{(2,-2)}^{(\mathrm{I} / \mathrm{II})}$ and $\mathcal{E}_{(3,-3)}^{(\mathrm{I} / \mathrm{II})}$ using the relations $\mathcal{E}_{(2,-1)}^{(\mathrm{I} / \mathrm{II})}=$ $\mathcal{E}_{(2,-2)}^{(\mathrm{I} / \mathrm{II})}+\omega$ and $\mathcal{E}_{(3,-2)}^{(\mathrm{I} / \mathrm{II})}=\mathcal{E}_{(3,-3)}^{(\mathrm{I} / \mathrm{II})}+\omega$, respectively.

\begin{tabular}{lrccc}
\hline \hline$(b, j)$ & $\mathcal{E}_{(b, j)}^{(\mathrm{I})}$ & $\mathcal{E}_{(b, j)}^{(\mathrm{II})}$ & $\Delta \mathcal{E}_{(b, j)}$ & $\left|\Delta \mathcal{E}_{(b, j)}\right| / \omega$ \\
\hline$(1,-1)$ & $-2.25 \times 10^{-3}$ & $-2.52 \times 10^{-3}$ & $-0.26 \times 10^{-3}$ & 0.05 \\
$(2,-1)$ & $-3.20 \times 10^{-3}$ & $-3.35 \times 10^{-3}$ & $-0.15 \times 10^{-3}$ & 0.03 \\
$(3,-2)$ & $0.45 \times 10^{-3}$ & $-0.10 \times 10^{-3}$ & $-0.55 \times 10^{-3}$ & 0.11 \\
$(3,-3)$ & $-4.55 \times 10^{-3}$ & $-5.10 \times 10^{-3}$ & $-0.55 \times 10^{-3}$ & 0.11 \\
$(4,-4)$ & & $-0.04 \times 10^{-3}$ & & \\
\hline
\end{tabular}

to be subject to the following selection rule: ${ }^{16} X_{(b, j),\left(b^{\prime}, j^{\prime}\right)}$ vanishes unless $\left|b-b^{\prime}\right|=$ odd numbers and $\left|j-j^{\prime}\right|=1$. This rule is evaluated from modeled SLs composed of square quantum wells with infinite barrier height. Thus, $X_{(b, j),\left(b^{\prime}, j^{\prime}\right)}$ is represented by $X \approx-2 F_{a c} d_{w} / \pi^{2}$ with $d_{w}$ as the well width of the quantum well, since all of the nonvanishing components are limited just to $\left|b-b^{\prime}\right|=1$ in the present case. A pair of $(b, j)$ and $\left(b^{\prime}, j^{\prime}\right)$ strongly coupled by the ac-Zener effect is connected by a solid line in Fig. 8; otherwise this effect is assumed to be negligibly weak. The red, blue, and black lines show the transitions mediated by the first-, second- and third-order ac-Zener couplings, respectively. The thick solid lines represent the couplings included in the calculation of Fig. 2, and the thin solid lines represent the rest of couplings that are excluded in this calculation.

The position of $\epsilon_{(b, j)}$ corresponds to the center of quasienergy band $(b, j)$ without the ac-ZT. Here, instead of calculating all of quasienergy bands, the quasienergy shift from $\epsilon_{(b, j)}$ is estimated in a nonperturbative manner, when the ac-ZT plays a decisive role. According to Fig. 8, the ac-Zener transition to which the most attention should be paid in the present estimate is the one between $(3,-3)$ and $(4,-4)$, since this serves as the most-significant first-order coupling and is excluded in the result of Fig. 2. Here, a sequence of the first-order couplings, that is, $(1,-1)-(2,-2)-(3,-3)-(4,-4)$, forms a dominant transition path. It is understood that the second-order transition between $(2,-2)$ and $(4,-4)$ and the third-order transition between $(1,-1)$ and $(4,-4)$ shown in Fig. 8 are mostly governed by this sequence.

The calculations are implemented in the case of $\alpha=60$ corresponding to $F_{a c}=1.16 \times 10^{-4}$, where $X \approx-2 \times 10^{-3}$; it is evident that the perturbation expansion can be applied no longer because of the ratio of $|X|$ to the representative energy separations of $\epsilon_{(b, j)}$ 's $\approx 1-2 \times 10^{-3}$ being of the order of unity. Here, two types of calculations are done by means of a standard diagonalization method. In the first one, three bases with quasienergy $\epsilon_{(b, j)}=(1,-1),(2,-2)$, and $(3,-3)$ are included, while in the second calculation one more basis of $(4,-4)$ is added to the first one; these two basis sets are termed Base I and Base II, respectively. The first calculation corresponds to the result of Fig. 2 with the lowest three minibands. On the other hand, the second one corresponds to the calculation with the lowest four minibands. The obtained results with Bases I and II are shown in Table I, where the eigenvalues are represented as $\mathcal{E}_{(b, j)}^{(\mathrm{I} / \mathrm{II})}$.

In spite of the calculation with Base I being quite simple, it is seen that the obtained result $\mathcal{E}_{(b, j)}^{(\mathrm{I})}$ approximately reproduces the band-center values of quasienergy lobes of $E(\alpha=60)$ shown in Fig. 2. The quasienergy difference of $\mathcal{E}_{(b, j)}^{(\mathrm{II})}$ from $\mathcal{E}_{(b, j)}^{(\mathrm{I})}$, that is, $\Delta \mathcal{E}_{(b, j)}^{(\mathrm{I} / \mathrm{II})} \equiv \mathcal{E}_{(b, j)}^{(\mathrm{II})}-\mathcal{E}_{(b, j)}^{(\mathrm{I})}$, and further, the error relative to the size of first Brillouin zone, that is, $\left|\Delta \mathcal{E}_{(b, j)}^{(\mathrm{I} / \mathrm{II})}\right| / \omega$, are shown in Table I. This implies relatively small effects of the SL miniband of $b=4$ on the lobes of $(1,-1)$ and $(2,-2)$, and thus this effect would cause just slight redshift of these lobes $(\leqslant 5 \%)$ within the Brillouin zone shown in Fig. 2, though the lobe of $(3,-3)$ is affected to a certain extent (accompanying the redshift by about 10\%). Because of such slight numerical errors in $\mathcal{E}_{(b, j)}^{(\mathrm{II})}$, there would still be room for further investigation of the rigorous proof of convergence of the quasienergy values. To conclude, it is considered that the $E(\alpha)$ 's shown in Fig. 2 are almost numerically convergent. Incidentally, the numerical convergence of the lobe of $(1, j)$ is most important, since spectral peaks of excess DOS attributed to the lobes of $(b \geqslant 2, j)$ tend to fade out with increasing $\alpha$, as shown in Fig. 3.

\footnotetext{
"hino@bk.tsukuba.ac.jp

${ }^{1}$ S. Kohler, J. Lehmann, and P. Hänggi, Phys. Rep. 406, 379 (2005).

${ }^{2}$ M. Grifoni and P. Hänggi, Phys. Rep. 304, 229 (1998), and references cited therein.

${ }^{3}$ S. D. Ganichev and W. Prettl, Intense Tetahertz Excitation of Semiconductors (Oxford University Press, Oxford, 2006).

${ }^{4}$ B. J. Keay, S. Zeuner, S. J. Allen, Jr., K. D. Maranowski, A. C. Gossard, U. Bhattacharya, and M. J. W. Rodwell, Phys. Rev. Lett. 75, 4102 (1995).

${ }^{5}$ K. W. Madison, M. C. Fischer, R. B. Diener, Qian Niu, and M. G. Raizen, Phys. Rev. Lett. 81, 5093 (1998).

${ }^{6}$ A. Eckardt, M. Holthaus, H. Lignier, Al Zenesini, D. Ciampini, O. Morsch, and E. Arimondo, Phys. Rev. A 79, 013611 (2009).

${ }^{7}$ S. Longhi, M. Marangoni, M. Lobino, R. Ramponi, P. Laporta, E. Cianci, and V. Foglietti, Phys. Rev. Lett. 96, 243901 (2006).
}

${ }^{8}$ G. Della Valle, M. Ornigotti, E. Cianci, V. Foglietti, P. Laporta, and S. Longhi, Phys. Rev. Lett. 98, 263601 (2007).

${ }^{9}$ A. Szameit, I. L. Garanovich, M. Heinrich, A. A. Sukhorukov, F. Dreisow, T. Pertsch, S. Nolte, A. Tünnermann, S. Longhi, and Y. S. Kivshar, Phys. Rev. Lett. 104, 223903 (2010).

${ }^{10}$ M. Sillanpää, T. Lehtinen, A. Paila, Y. Makhlin, and P. Hakonen, Phys. Rev. Lett. 96, 187002 (2006).

${ }^{11}$ N. Tsuji, T. Oka, and H. Aoki, Phys. Rev. B 78, 235124 (2008).

${ }^{12}$ D. H. Dunlap and V. M. Kenkre, Phys. Rev. B 34, 3625 (1986).

${ }^{13}$ M. Holthaus, Phys. Rev. Lett. 69, 351 (1992).

${ }^{14}$ J. Zak, Phys. Rev. Lett. 71, 2623 (1993).

${ }^{15}$ J. Rotvig, A.-P. Jauho, and H. Smith, Phys. Rev. Lett. 74, 1831 (1995); Phys. Rev. B 54, 17691 (1996).

${ }^{16} \mathrm{M}$. Holthaus and D. W. Hone, Philos. Mag. B 74, 105 (1996). 
${ }^{17}$ K. I. Hino, K. Yashima, and N. Toshima, Phys. Rev. B 71, 115325 (2005).

${ }^{18}$ M. Glück, A. R. Kolovsky, and H. J. Korsch, Phys. Rep. 366, 103 (2002).

${ }^{19}$ A. Kukuu, T. Amano, T. Karasawa, N. Maeshima, and K. I. Hino, Phys. Rev. B 82, 115315 (2010).

${ }^{20}$ M. Gavrila, in Atoms in Intense Laser Fields, edited by M. Gavrila (Academic, New York, 1992), p. 435, and references cited therein.

${ }^{21}$ W. C. Henneberger, Phys. Rev. Lett. 21, 838 (1968).

${ }^{22}$ K. Yajima, Commun. Math. Phys. 87, 331 (1982).

${ }^{23}$ J. Zak, Phys. Rev. Lett. 20, 1477 (1968).

${ }^{24}$ J. E. Avron, J. Zak, A. Grossman, and L. Gunther, J. Math. Phys. 18, 918 (1977).

${ }^{25}$ H. Schneider, H. T. Grahn, K. von Klizting, and K. Ploog, Phys. Rev. Lett. 65, 2720 (1990).

${ }^{26}$ M. Nakayama, I. Tanaka, H. Nishimura, K. Kawashima, and K. Fujiwara, Phys. Rev. B 44, 5935 (1991).

${ }^{27}$ K. I. Hino and N. Toshima, Phys. Rev. B 71, 205326 (2005), and references cited therein.

${ }^{28}$ F. T. Smith, Phys. Rev. 118, 349 (1960).

${ }^{29}$ R. Balian and C. Bloch, Ann. Physics (NY) 60, 401 (1970).

${ }^{30}$ J. L. Kinsey, Chem. Phys. Lett. 8, 349 (1971).

${ }^{31}$ M. Desouter-Lecomte and X. Chapuisat, Phys. Chem. Chem. Phys. 1, 2635 (1999), and references cited therein.

${ }^{32}$ J. Purvis, M. Dörr, M. Terao-Dunseath, C. J. Joachain, P. G. Burke, and C. J. Noble, Phys. Rev. Lett. 71, 3943 (1993), and references cited therein.

${ }^{33}$ P. G. Burke, R-Matrix Theory of Atomic Collisions: Application to Atomic, Molecular and Optical Processes (Springer-Verlag, Berlin, 2011), Chap. 9.

${ }^{34}$ S.-I. Chu and D. A. Telnov, Phys. Rep. 390, 1 (2004).
${ }^{35}$ R. M. Potvliege and R. Shakeshaft, in Atoms in Intense Laser Fields, edited by M. Gavrila (Academic, New York, 1992), p. 373, and references cited therein.

${ }^{36}$ Y. K. Ho, Phys. Rep. 99, 1 (1983).

${ }^{37}$ N. Moiseyev, Phys. Rep. 302, 212 (1998).

${ }^{38}$ B. R. Junker, Adv. At. Mol. Phys. 18, 207 (1982).

${ }^{39}$ W. P. Reinhardt, Annu. Rev. Phys. Chem. 33, 223 (1982).

${ }^{40}$ N. Maeshima and K. Hino, Comput. Phys. Commun. 183, 8 (2012).

${ }^{41}$ N. Maeshima and K. I. Hino, Phys. Rev. B 85, 205305 (2012).

${ }^{42}$ R. G. Newton, Scattering Theory of Waves and Particles, 2nd ed. (Springer-Verlag, New York, 1982), Chap. 12.

${ }^{43}$ B. W. Shore, Rep. Mod. Phys. 39, 439 (1967).

${ }^{44}$ R. B. Liu and B. F. Zhu, Phys. Rev. B 59, 5759 (1999).

${ }^{45}$ M. V. Ammosov, N. B. Delone, and V. P. Krainov, Sov. Phys. JETP 64, 1191 (1986).

${ }^{46}$ U. Fano, Phys. Rev. 124, 1866 (1961).

${ }^{47}$ S. N. Elaydi, Discrete Chaos, 2nd ed. (Chapman \& Hall/CRC, Boca Raton, 2008), Chap. 4.

${ }^{48}$ H. Hirori, M. Nagai, and K. Tanaka, Phys. Rev. B 81, 081305(R) (2010).

${ }^{49}$ M. M. Dignam, Phys. Rev. B 59, 5770 (1999).

${ }^{50}$ T. Meier, G. von Plessen, P. Thomas, and S. W. Koch, Phys. Rev. B 51, 14490 (1995).

${ }^{51}$ J. M. Lachaine, M. Hawton, J. E. Sipe, and M. M. Dignam, Phys. Rev. B 62, R4829 (2000).

${ }^{52}$ T. Meier, H. J. Kolbe, A. Thränhardt, G. Weiser, P. Thomas, and S. W. Koch, Physica E 7, 267 (2000).

${ }^{53}$ D. Wang, A. Zhang, L. Yang, and M. M. Dignam, Phys. Rev. B 77, 115307 (2008).

${ }^{54} \mathrm{H}$. Haug and S. W. Koch, Quantum Theory of the Optical and Electronic Properties of Semiconductors, 5th ed. (World Scientific, Singapore, 2009), Chap. 12. 